\title{
1 Spin-polarized Oxygen Evolution Reaction under Magnetic Field
}

2 Xiao Ren ${ }^{1,2}$, Tianze $\mathrm{Wu}^{1,2,3}$, Yuanmiao $\mathrm{Sun}^{2}$, Yan $\mathrm{Li}^{1}$, Guoyu Xian ${ }^{1}$, Xianhu Liü ${ }^{4}$, Chengmin

3 Shen $^{1}$, Jose Gracia ${ }^{5}$, Hong-Jun Gao ${ }^{1}$, Haitao Yang ${ }^{1 *}$, Zhichuan J. Xu $u^{2,3,6 *}$

4

$5 \quad{ }^{1}$ Beijing National Laboratory for Condensed Matter Physics and Institute of Physics, Chinese

6 Academy of Science, P.O.Box 603, Beijing, 100190, China;

$7 \quad{ }^{2}$ School of Material Science and Engineering, Nanyang Technological University, 50 Nanyang

8 Avenue, Singapore 639798, Singapore;

$9 \quad{ }^{3}$ Solar Fuels Laboratory, Nanyang Technological University, 50 Nanyang Avenue, Singapore

10 639798, Singapore;

$11{ }^{4}$ Key Laboratory of Advanced Material Processing \& Mold (Zhengzhou University), Ministry

12 of Education, Zhengzhou 450002, China;

$13{ }^{5}$ MagnetoCat SL, General Polavieja 9 3I, 03012 Alicante, Spain;

$14{ }^{6}$ Energy Research Institute @ Nanyang Technological University, 50 Nanyang Avenue,

15 Singapore 639798, Singapore.

16 E-mails: xuzc@ntu.edu.sg; htyang@iphy.ac.cn 


\section{Abstract}

20

The oxygen evolution reaction (OER) is the bottleneck that limits the energy efficiency of water-splitting. The process involves four electrons' transfer and the generation of triplet state $\mathrm{O}_{2}$ from singlet state species $\left(\mathrm{OH}^{-}\right.$or $\left.\mathrm{H}_{2} \mathrm{O}\right)$. Recently, explicit spin selection was described as a possible way to promote OER in alkaline conditions, but the specific spin-polarized kinetics remains unclear. Here, we report that by using ferromagnetic ordered catalysts as the spin polarizer for spin selection under a constant magnetic field, the OER can be enhanced. However, it does not applicable to non-ferromagnetic catalysts. We found that the spin polarization occurs at the first electron transfer step in OER, where coherent spin exchange happens between the ferromagnetic catalyst and the adsorbed oxygen species with fast kinetics, under the principle of spin angular momentum conservation. In the next three electron transfer steps, as the adsorbed $\mathrm{O}$ species adopt fixed spin direction, the OER electrons need to follow the Hund rule and Pauling exclusion principle, thus to carry out spin polarization spontaneously and finally lead to the generation of triplet state $\mathrm{O}_{2}$. Here, we showcase spin-polarized kinetics of oxygen evolution reaction, which gives references in the understanding and design of spin-dependent catalysts. 


\section{Introduction}

The sluggish kinetics of oxygen evolution reaction (OER) is a major cause for the low efficiency in techniques such as solar water splitting, ${ }^{1}$ rechargeable metal-air batteries, ${ }^{2}$ regenerative fuel cells, ${ }^{3}$ and water electrolysis. ${ }^{4,5}$ Exploring better catalysts for OER has become increasingly attractive in recent years. Non-precious 3d-transition metal oxides (TMOs), such as Fe-, Co-, and Ni-based oxides, are promising cost-effective catalysts. ${ }^{6,7}$ Their catalytical activities are tunable as the diversity in oxides families affords numerous freedoms to tailor their physicochemical properties. Sabatier's principle, which qualitatively describes that the optimized catalytic activity when adsorbed species bind to the catalytic surface neither too strongly nor too weakly, led to the fundamental understanding of OER mechanisms and guided the subsequent design of highly active catalysts. ${ }^{8,9}$ This principle was further supported by the findings that the OER activities of transition metal oxides correlate strongly with the $\mathrm{e}_{\mathrm{g}}$ occupancy, which is related to the binding strength between the metal and the oxygen species. ${ }^{10-}$ ${ }^{12}$ Some exceptions have been found not well fitted with the $e_{\mathrm{g}}$ theory, which is partially resulted by the diverse and complicated magnetism in TMOs family. ${ }^{13-16}$ Besides, the produced $\mathrm{O}_{2}$ is in triplet ground state, where the frontier $\pi^{*}$ orbitals are occupied by two electrons with parallel alignment. In contrast, the ground spin state of reactant $\mathrm{OH}^{-} / \mathrm{H}_{2} \mathrm{O}$ is singlet with all paired electrons. ${ }^{17,18}$ The singlet states of the oxygen molecule were reported at an energy level of at least $\sim 1 \mathrm{eV}$ higher than its triplet state. ${ }^{18,19}$ Thus, the magnetism of TMOs, related to the spin polarization, should be influential on the kinetics of OER. ${ }^{20,21}$ It is reasonable to consider that the active sites with suitable thermodynamic paths for OER should allow a way to facilitate the spin alignment in the product. As suggested by recent theoretical studies by J. Gracia, ${ }^{22-24}$ the spin-polarized electrons in catalysts promote the generation of parallel spin aligned oxygen by quantum spin-exchange interactions (QSEI), which further promote the OER kinetics. Therefore, facilitating the spin polarization should be an effective strategy for improving OER efficiency. Ron Naaman and co-works reported that the application of the chiral-induced spin selectivity effect to product the polarized electron. This spin polarization transferred is the origin of a more efficient oxidation process in which oxygen is formed in its triplet ground state. ${ }^{25-27}$ It has been pointed out by J. Gracia et al. that theoretically photosystem II can act as 
a spin-controlling gate to govern the charge and spin transport during the OER process, ${ }^{28}$ which offers a favoured thermodynamic path for $\mathrm{O}_{2}$ evolution. Besides the extrinsic spin polarizer, the ordered magnetic moment structure in ferromagnetic materials can create intrinsic spin filtering for highly spin-polarized electrons. The spin filtering effect originates from the exchange splitting of the energy levels in the conduction band of a magnetic insulator. ${ }^{29}$ Most recently, José Ramón Galán-Mascarós et al. reported an external magnetic field, applied by a permanent magnet, enhances the OER activity of magnetic oxides in alkaline. ${ }^{30}$ It opens a new strategy to manipulate the spin polarization in magnetic oxide catalysts for promoting the OER and encourages more detailed studies to understand how the magnetic field induced spin polarization affects the OER process.

In this work, we report an investigation on the key kinetics change on the ferromagnetic $\mathrm{CoFe}_{2} \mathrm{O}_{4}$ catalyst under the magnetic field. The ferromagnetic $\mathrm{CoFe}_{2} \mathrm{O}_{4}$ catalyst works as a spin polarizer under the magnetic field. We have found that the spin-polarized kinetics of OER starts at the first electron transfer step, where ferromagnetic exchange happens between the ferromagnetic catalysts and the adsorbed oxygen species (reactants) under the principle of spin angular momentum conservation. Without the magnetic field, the Tafel slope of $\mathrm{CoFe}_{2} \mathrm{O}_{4}$ is identical and equal to circa $120 \mathrm{mV} /$ decade, which indicates the first electron transfer step is rate-determining step (RDS) and no electron transfer occurring before the RDS. Under the magnetic field, the Tafel slope decreases to circa $90 \mathrm{mV} /$ decade, indicating the number of electron transfer is $\sim 0.5$ and a mixed RDS involving the first electron transfer step and second steps. Such a phenomenon cannot be observed in the catalysts without ferromagnetic orderings under the same condition. The results indicate that the key step of spin-polarized OER is the first electron transfer step in OER, where the spin-polarized process via exchange hopping can be facilitated under the magnetic field. As a consequence, the first electron transfer is no longer the RDS. After a facilitated spin-polarized ferromagnetic exchange of electrons, the adsorbed O species will overall settle on the fixed spin direction. Due to the Hund Rule and Pauli Exclusion Principle, the follow-up electrons' transfer needs to carry out spin polarization spontaneously and finally lead to the generation of triplet state oxygen. Overall, we showcase 
the key kinetics information in OER under a magnetic field, which influences the micro- and macroscopic spin-polarization and spin-transport. This finding will be helpful for further development of magnetic-field assisted OER-enhancing strategy and related catalysts.

\section{Results and discussion}

\section{Magnetic and electrochemical characterizations}

We begin with the discussion of the magnetic properties of the employed catalysts, $\mathrm{CoFe}_{2} \mathrm{O}_{4}$, $\mathrm{Co}_{3} \mathrm{O}_{4}$, and $\mathrm{IrO}_{2}$. The study will determine the suitable magnetic field and weather a global aligned magnetic moment can be achieved. The study of magnetic property reveals the optimal strength of the applied magnetic field for the alignment of the magnetic moment in ferromagnetic $\mathrm{CoFe}_{2} \mathrm{O}_{4}$. The $\mathrm{CoFe}_{2} \mathrm{O}_{4}$ and $\mathrm{Co}_{3} \mathrm{O}_{4}$ were prepared by a modified solid-state chemistry method as previously reported. ${ }^{11} \mathrm{X}$-ray powder diffraction characterization was performed to confirm their crystal structures. The diffraction patterns match well with the standard patterns without impurity peak found (Supplementary Figure 1 and Supplementary Table 1). As depicted in Figure 1a, $\mathrm{CoFe}_{2} \mathrm{O}_{4}$ gives a hysteresis loop in an enlarged manner, indicating a room-temperature ferromagnetic behavior with a saturation magnetization (Ms) of $44 \mathrm{emu} \cdot \mathrm{g}^{-1}$. The $\mathrm{Co}_{3} \mathrm{O}_{4}$ and $\mathrm{IrO}_{2}$ samples with tiny susceptibility $(\chi)$ of $3.07 \times 10^{-5}$ and $0.51 \times 10^{-}$ ${ }^{6}$, respectively, at $300 \mathrm{~K}$ show antiferromagnetic or paramagnetic behaviors, respectively. The detailed magnetic data are summarized in Supplementary Table 1. The cyclic voltammetry (CV) of those catalysts were then measured with and without a constant magnetic field of $10000 \mathrm{Oe}$ under alkaline condition (see Methods for details). As shown in Figure 1b, c, and d, the OER performance of the ferromagnetic $\mathrm{CoFe}_{2} \mathrm{O}_{4}$ is promoted obviously under the magnetic field while the changes in non-ferromagnetic catalysts $\mathrm{Co}_{3} \mathrm{O}_{4}$ and $\mathrm{IrO}_{2}$ are negligible. When a strong enough magnetic field (higher than the coercivity) is applied to a ferromagnetic material, the magnetic moment will (macroscopically) align along with the direction of the external magnetic field. The ferromagnetic (long-) ordered material as spin polarizer is an extended selective spinfilter for electron transfer during catalysis. The generation process of polarized electrons has been illustrated in Figure 1e. 
122 It should be noticed that the use of magnetic fields in water electrolysis has been studied in the 123 past, ${ }^{31-36}$ in which the mass transport in the electrochemical process was found to be affected 124 by the Lorentzian movement, i.e. the diffusion of regents and the release of the generated gas 125 bubbles are promoted. However, in this study, some evidence has excluded such effect from mass transport as a main contributor to OER enhancement under the magnetic field. First, the 127 improvement was not observed on non-ferromagnetic catalysts $\mathrm{Co}_{3} \mathrm{O}_{4}$ and $\mathrm{IrO}_{2}$ with the effect 128 of Lorentzian movement on mass transport. Second, we also tested the OER performance of $129 \mathrm{Co}(\mathrm{acac})_{2}$ and $\mathrm{Fe}(\mathrm{acac})_{3}$ with and without a constant magnetic field (as shown in Supplementary 130 Figure 2). Nearly no difference can be observed. It also should be noted that $\mathrm{OH}^{-}$and $\mathrm{H}_{3} \mathrm{O}^{+}$in 131 aqueous solution do not move physically, but by sequential proton transfer, known as Grotthuss mechanisms (Supplementary Figure 3). That means the influence of Lorentz force on the 133 physical movement of ions $\mathrm{OH}^{-}$or $\mathrm{H}_{3} \mathrm{O}^{+}$is negligible ${ }^{37}$. Thus, the effect from the mass transport 134 under the external magnetic field should have little contribution to the observed OER 135 enhancement of the ferromagnetic $\mathrm{CoFe}_{2} \mathrm{O}_{4}$. Besides, the electrical resistance of magnetic 136 materials can be affected by the magnetization, which is through the scattering of electrons on 137 the magnetic lattice of the crystal. ${ }^{38-40}$ However, the difference in the conductivity at room 138 temperature under 10000 Oe is about $3 \%$ for insulator $\mathrm{CoFe}_{2} \mathrm{O}_{4}$ with $3.86 \times 10^{-5} \mathrm{~S} / \mathrm{m},{ }^{40-42}$ which 139 does not cause a significant difference in the electrode's conductivity. This is because acetylene 140 black carbon $(\mathrm{AB})$ with $500 \mathrm{~S} / \mathrm{m}$ as a conductive mediator is mixed with those oxide catalysts 141 for their application as the electrode, ${ }^{43}$ which dominants the electron conduction. 


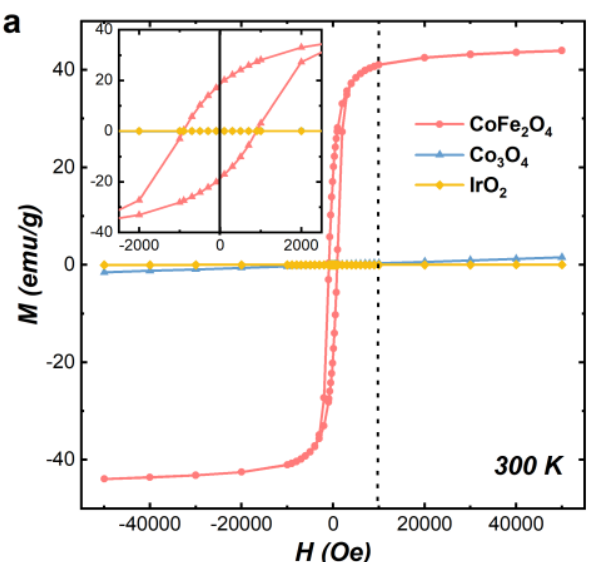

e
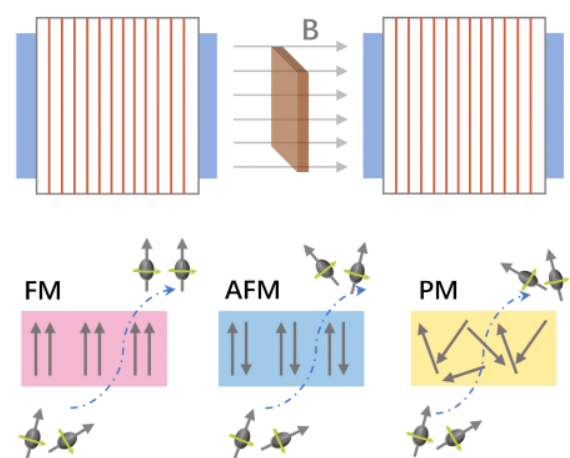
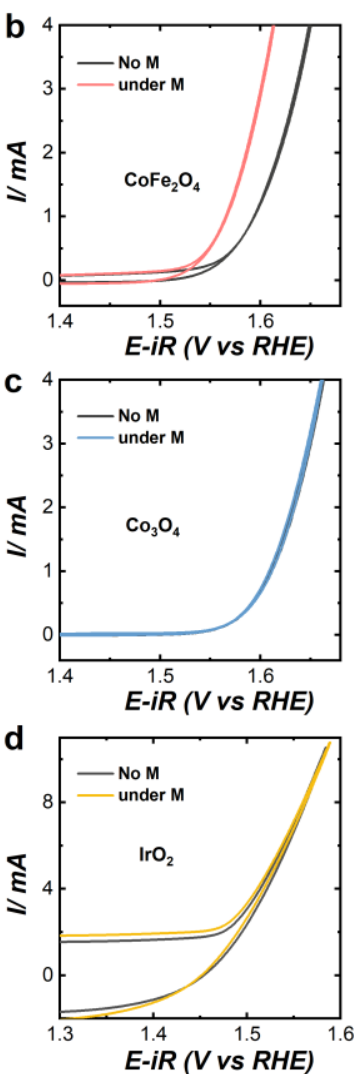
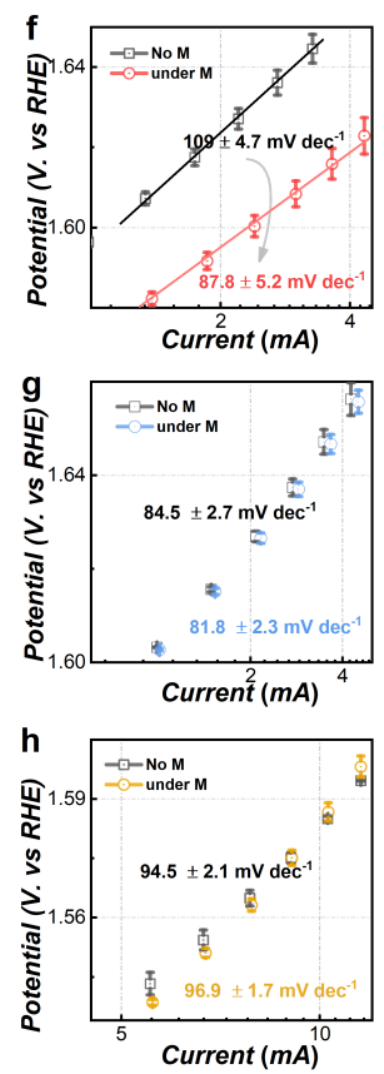

Figure 1 | Spin polarization promotes OER. (a) Magnetic hysteresis loops of $\mathrm{CoFe}_{2} \mathrm{O}_{4}, \mathrm{Co}_{3} \mathrm{O}_{4}$, and $\mathrm{IrO}_{2}$ powders at room temperature $(300 \mathrm{~K})$ and the magnified graph inset in the top left of this panel. (Here, $\mathrm{IrO}_{2}$ powder is a commercial catalyst (bulk, Premetek). Cyclic voltammetry (CV) of $\mathrm{CoFe}_{2} \mathrm{O}_{4}(\mathbf{b}), \mathrm{Co}_{3} \mathrm{O}_{4}(\mathbf{c})$, and $\mathrm{IrO}_{2}(\mathbf{d})$ catalysts at a scan rate of $10 \mathrm{mV} / \mathrm{s}$ in $\mathrm{O}_{2}$-saturated $1 \mathrm{M} \mathrm{KOH}$ with and without a constant magnetic field (10000 Oe). (e) The schematic of the generation of the polarized electron under a constant magnetic field. The Tafel plots of $\mathrm{CoFe}_{2} \mathrm{O}_{4}$, (f) $\mathrm{Co}_{3} \mathrm{O}_{4}(\mathbf{g})$, and $\mathrm{IrO}_{2}$ (h) catalysts with and without a constant magnetic field (10000 Oe). The error bar represents three independent tests.

\section{No surface restructuration in OER}

It is generally recognized that some Co-based perovskites and spinels undergo operando surface reconstruction to form active Co (oxy) hydroxides in alkaline conditions to promote OER. ${ }^{44-46}$ In our case, there are no changes in OER performance of $\mathrm{CoFe}_{2} \mathrm{O}_{4}$ during $\mathrm{CA}$ tests in $1 \mathrm{M} \mathrm{KOH}$ for $1 \mathrm{~h}$ shown in Supplementary Figure 4, indicating $\mathrm{CoFe}_{2} \mathrm{O}_{4}$ is stable without noticed surface reconstruction during the OER process. The high-resolution transmission electron microscope 
(HRTEM) was further used to rule out the possible interference from surface reconstruction of catalysts during the OER. It has been found that the spinel crystal structure of $\mathrm{CoFe}_{2} \mathrm{O}_{4}$ remained after the electrochemical treatment (Supplementary Figure 5), which is consistent with what has been reported previously. ${ }^{47}$ The aberration-corrected STEM provides direct atomic imaging and confirms that the well-crystalline feature reserved from the surface to bulk (Figure 2a, b). The HADDF line profile shows the same bond length of Co-O in bulk and surface, which verifies no surface reconstruction (Figure 2c). Raman technique was then performed to study the inhomogeneity evolution in the near-surface region. The Raman spectra of cubic structures (Fd-3m) $\mathrm{CoFe}_{2} \mathrm{O}_{4}$ before and after OER are presented in Figure $2 \mathrm{~d}$. In the top curve, peak maxima at 603 and $666 \mathrm{~cm}^{-1}$ are due to $\mathrm{A}_{1 \mathrm{~g}}$ symmetry involving symmetric stretching of oxygen atom with respect to the metal ions in tetrahedral sites. The other lowfrequency phonon modes are due to metal ions involved in octahedral sites, i.e., $\mathrm{E}_{\mathrm{g}}$ and $\mathrm{T}_{\mathrm{gg}}$. The assignment of these phonon modes was carried out in accordance with the literature. ${ }^{48}$ After electrochemical treatment (bottom curve), no changes in the vibrational modes were observed, which proves once again that there is no surface reconstruction in OER. X-ray photoelectron spectroscopy (XPS) was also performed to study the surface chemical states of $\mathrm{CoFe}_{2} \mathrm{O}_{4}$ before and after the OER. As shown in Supplementary Figure 6, the XPS survey spectra confirm the coexistence of $\mathrm{Co}, \mathrm{Fe}$, and $\mathrm{O}$ in the samples. Figure $2 \mathrm{e}$ shows the $2 \mathrm{p}$ orbital of $\mathrm{Fe}, \mathrm{Co}$, and 1s orbital of O. The XPS of Fe $2 p$ core level presents two pairs of peaks: $\mathrm{Fe}^{3+} 2 \mathrm{p}_{3 / 2}$ at about 710.7 $\mathrm{eV}$ and $713.2 \mathrm{eV} ; \mathrm{Fe}^{3+} 2 \mathrm{p}_{1 / 2}$ at about $724.1 \mathrm{eV}$ and $726.2 \mathrm{eV}$. The doublets in samples can be ascribed to $\mathrm{Fe}^{3+}$ in octahedral sites and $\mathrm{Fe}^{3+}$ in tetrahedral sites, respectively. The two peaks of Co $2 p$ with the binding energy of 780.1 and $782.0 \mathrm{eV}$ are ascribed to $\mathrm{Co}^{2+}$ ions in octahedral sites and $\mathrm{Co}^{2+}$ ions in tetrahedral sites. The main peaks of $\mathrm{O} 1 \mathrm{~s}$ at $529.6 \mathrm{eV}$ are recognized as oxygen ions, which are all associated with a "-2" formal charge. ${ }^{49}$ Compared to the spectra before and after the OER, these peaks remain unchanged in location, indicating no surface reconstruction. 

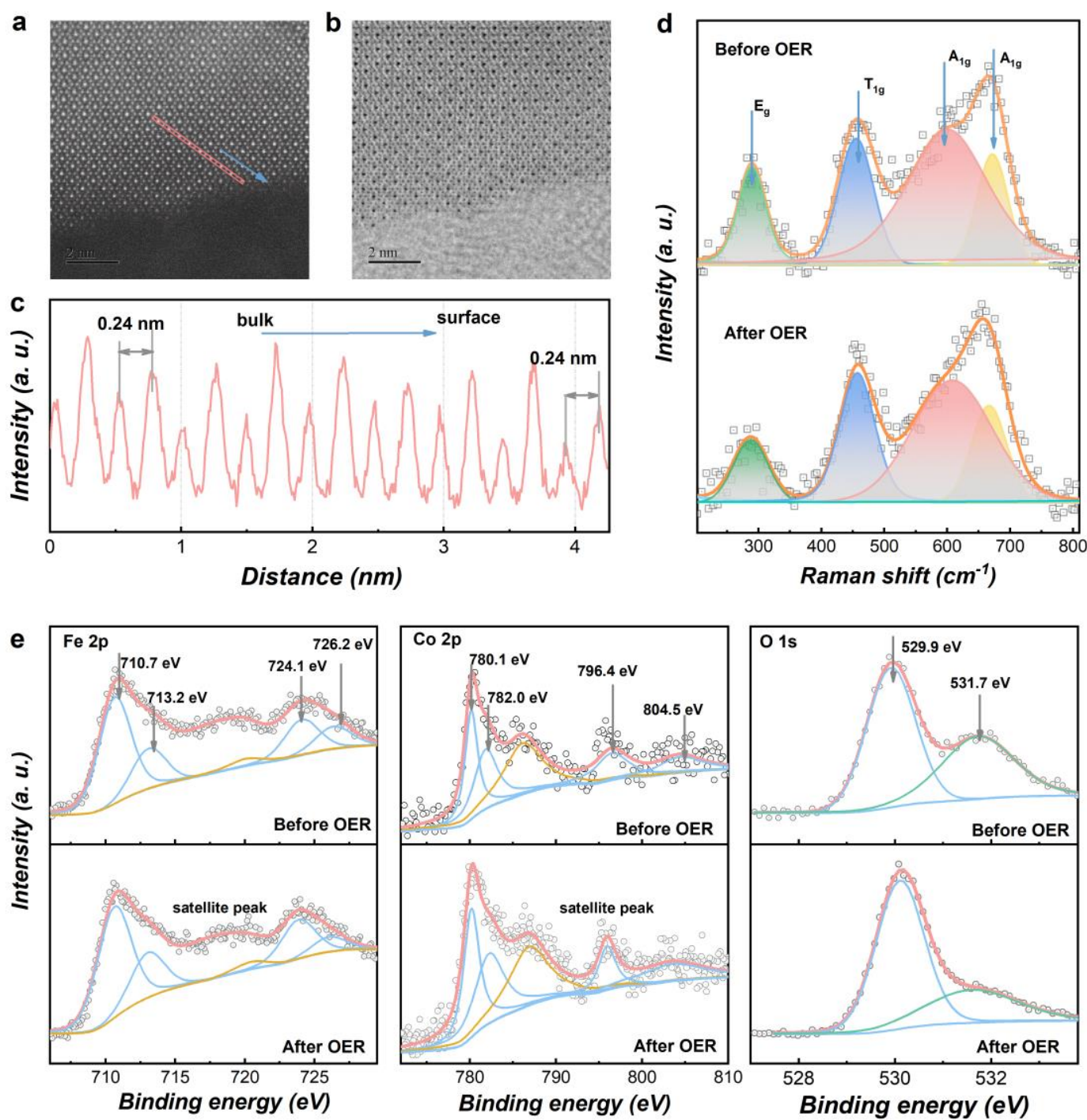

Figure 2 | No surface restructuration on $\mathrm{CoFe}_{2} \mathrm{O}_{4}$. HADDF (a) and ABF (b) images of $\mathrm{CoFe}_{2} \mathrm{O}_{4}$ after OER. The line profiles of HADDF (c) acquired at the pink line rectangular zone. (d) Raman spectra of $\mathrm{CoFe}_{2} \mathrm{O}_{4}$ before and after OER. (e)The Fe 2p, Co 2p and O 1s XPS spectra results of $\mathrm{CoFe}_{2} \mathrm{O}_{4}$ before and after OER.

\section{Spin-polarized Kinetics of OER}

192 Oxygen evolution reaction is authenticated a four-step reaction with each step accompanied by an electron transfer as shown in the top of Figure 4a. The Tafel plots are widely regarded as a generalized kinetics theory for electron transfer reactions. The Tafel equation presents the relationship between the Tafel slope and the exchange current density: 


$$
\eta=-\frac{2.303 R T}{\alpha F} * \log i_{0}+\frac{2.303 R T}{(\alpha+n) F} * \log i
$$

where the Tafel slope equals to $2.303 \mathrm{RT} /[(\alpha+n) \mathrm{F}]$ ( $\mathrm{i}_{0}$ is the exchange current density, $\mathrm{R}$ is the universal gas constant, $\mathrm{T}$ is the absolute temperature, $\mathrm{F}$ is the Faraday constant, $\mathrm{n}$ is the number of electrons transferred before RDS, and $\alpha$ is the charge transfer coefficient and usually assumed to be 0.5$).{ }^{50,51}$ Ideally, the Tafel slope tells the information of reaction kinetics. For example, the Tafel slope is $120 \mathrm{mV} \cdot \mathrm{dec}^{-1}$, which indicates the first electron transfer step is the RDS because there is no electron transfer before the RDS. If the second step is the RDS, the Tafel slope will decrease to $40 \mathrm{mV} \cdot \mathrm{dec}^{-1}$ with an electron transfer number of 1 . The change of the Tafel slope is often reputed as an indication of the change of reaction mechanism. As shown in Figure 1f, the Tafel slope of $\mathrm{CoFe}_{2} \mathrm{O}_{4}$ is about $109 \pm 4.7 \mathrm{mV} \cdot \mathrm{dec}^{-1}$ and that indicates the first electron transfer from the adsorbed $\mathrm{OH}^{-}$is the RDS without the magnetic field. But, after applying a constant magnetic field, the Tafel slope decreases to circa $87.8 \pm 5.2 \mathrm{mV} \cdot \mathrm{dec}^{-1}$, indicating the number of electron transfer is about 0.5 and a mixed RDS involving the first electron transfer step and second steps. Furthermore, we have carried out OER measurements of $\mathrm{CoFe}_{2} \mathrm{O}_{4}, \mathrm{Co}_{3} \mathrm{O}_{4}$, and $\mathrm{IrO}_{2}$ under different temperatures as shown in Figure 3a. We first noted that the OER performance of catalysts is getting better as the reaction temperature increases. This is probably because that the rate constant of the reaction will increase as the reaction temperature increases, which can promote this reaction based on the transition state theory ${ }^{52}$. More importantly, the OER performance of the ferromagnetic $\mathrm{CoFe}_{2} \mathrm{O}_{4}$ is promoted under the magnetic field at various temperature. However, the positive influence of the magnetic field on the OER performance of $\mathrm{CoFe}_{2} \mathrm{O}_{4}$ is decreased as the reaction temperature increases. The corresponding Tafel slopes are shown in Figure 3b. At room temperature, the Tafel slope of $\mathrm{CoFe}_{2} \mathrm{O}_{4}$ is about $106 \mathrm{mV} \cdot \mathrm{dec}^{-1}$ without the magnetic field. After applying a constant field, the Tafel slope decreases to circa $82.8 \mathrm{mV} \cdot \mathrm{dec}^{-1}$. As the temperature increases, the positive influence of the magnetic field became not that remarkable. This is because the arrangement of magnetic moments of catalyst will be thermally disturbed. The ferromagnetic ordering in the catalyst gets disturbed and thus a certain degree of demagnetization at high temperature occurs, which lead to the decreased influence of the magnetic field on OER. We also note that the Tafel 
slope of $\mathrm{CoFe}_{2} \mathrm{O}_{4}$ have a slight favorable change as temperature increases, which maybe because the interaction between two M-O unites mechanism occurs at high temperature. ${ }^{53,54}$ Thus, the key step in spin-polarized OER is the first electron transfer step in FM $\mathrm{CoFe}_{2} \mathrm{O}_{4}$, where the adsorbed $\mathrm{OH}^{-}$is difficult to deprotonate and transfer the electron. However, the change of Tafel slopes was not observed in the non-ferromagnetic catalysts under the same condition.
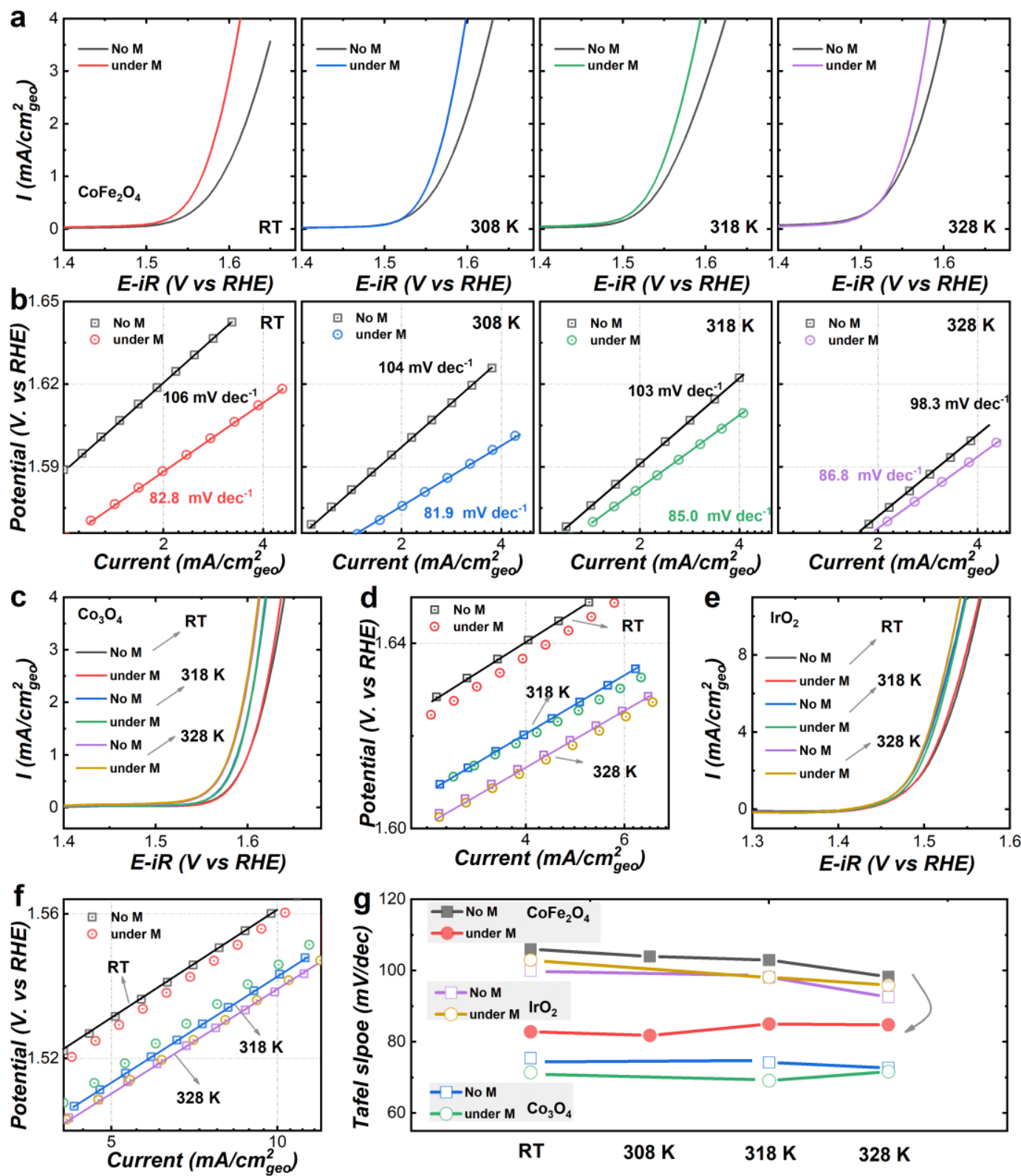

Figure 3 | OER under the different temperature. (a) LSV curves of $\mathrm{CoFe}_{2} \mathrm{O}_{4}$ at a scan rate of $10 \mathrm{mV} / \mathrm{s}$ in $\mathrm{O}_{2}$-saturated $1 \mathrm{M} \mathrm{KOH}$ with and without a constant magnetic field (10000 Oe) 
under the different temperatures (room temperature (RT): $\sim 303 \mathrm{~K}, 308 \mathrm{~K}, 318 \mathrm{~K}$ and $328 \mathrm{~K}$ ). The corresponding Tafel plots are shown in (b). (c) LSV curves of $\mathrm{Co}_{3} \mathrm{O}_{4}$ with and without a constant magnetic field (10000 Oe) under the different temperatures (room temperature (RT): $\sim 303 \mathrm{~K}, 313 \mathrm{~K}$ and $323 \mathrm{~K})$. The corresponding Tafel plots are shown in (d). (e) LSV curves of $\mathrm{IrO}_{2}$ with and without a constant magnetic field $(10000 \mathrm{Oe})$ under the different temperatures (room temperature (RT): $\sim 303 \mathrm{~K}, 313 \mathrm{~K}$ and $323 \mathrm{~K}$ ). The corresponding Tafel plots are shown in (f). Tafel slopes at various temperatures are summarized in $(\mathbf{g})$.

The electron transfer at the catalytic interface depends on the transition probability, which is associated with the wavefunction integral between $\mathrm{OH}^{-}$and the active site. As revealed by our previous work, the octahedral sites are mainly responsible to the OER ${ }^{55}$. The extended X-ray absorption fine structure (EXAFS) showed the perfect inverse spinel structure of $\mathrm{CoFe}_{2} \mathrm{O}_{4}$ (Supplementary Figure 7). The $\mathrm{Fe}^{3+}$ ions distribute equally in octahedral and tetrahedral sites and $\mathrm{Co}^{2+}$ ions distribute in octahedral sites. We further calculated the effective magnetic moment $\left(\mu_{e f f}\right)$ of $\mathrm{CoFe}_{2} \mathrm{O}_{4}$ to be about $3.44 \mu_{\mathrm{B}}$ by Curie-Weiss fitting (Supplementary Figure 8). The $\mu_{e f f}$ for $\mathrm{CoFe}_{2} \mathrm{O}_{4}$ is very close to the idea value of the inverse spinel. ${ }^{56}$ Thus, the $\mathrm{Co}^{2+}$ ions in octahedral sites contribute to the effective ferromagnetic moment. Those results are consistent in previous experimental work. ${ }^{57}$ Considering that only Co in octahedral sites contribute the effective magnetic moment, the magnetic field enhanced OER should mainly happen on the Co sites. Thus, we studied the Co sites as the active sites in this work. For a ferromagnetic (FM) catalyst, the orbitals of the FM oxides create an intrinsically degenerate spin-polarized metallic state that optimizes the wavefunction based on the inter-atomic reduction of the electron-electron repulsion. DFT calculations were performed to explore the diffierent elctron structure of $\mathrm{CoFe}_{2} \mathrm{O}_{4}$ under an applied magnetic field (The computational details are shown in the Supplementary Information). As shown in the projected density of states (PDOS) of $\mathrm{CoFe}_{2} \mathrm{O}_{4}$ (Figure 4a), the $3 \mathrm{~d}-2 \mathrm{p}$ hybridization of the $\mathrm{CoFe}_{2} \mathrm{O}_{4}$ become stronger after aligning the spins. As well, compared to the $\mathrm{CoFe}_{2} \mathrm{O}_{4}$ with anti-parallel couplings, the $\mathrm{CoFe}_{2} \mathrm{O}_{4}$ with spin alignment has a higher spin density on the oxygen atoms (Figure $4 \mathrm{~b}$ ). The 
calculation indicates that the magnetic moment of the ligand hole in $\mathrm{CoFe}_{2} \mathrm{O}_{4}$ is $0.059 \mu \mathrm{B}$ without spin alignment and is $0.188 \mu \mathrm{B}$ with spin alignment, which indicates a FM ligand hole in $\mathrm{CoFe}_{2} \mathrm{O}_{4}$. A concomitant increment of the $3 \mathrm{~d}-2 \mathrm{p}$ hybridization associate with FM ligand holes will facilitate spin-selected charge transport and optimize the kinetics of the spin-charge transfer in the three-phase interface. ${ }^{43,} 58$ Thus, the dominant FM exchange between the ferromagnetic catalyst and the adsorbed oxygen species (reactants) will happen (Figure $4 \mathrm{c}$ and Supplementary Figure 9) with smaller electron-electron repulsion, which induce spindependent conductivity and decrease the rate-limiting bonding energies, making that the first electron transfer is no longer the RDS. We further prepared the Pourbaix diagram of $\mathrm{CoFe}_{2} \mathrm{O}_{4}$ as shown in Figure 4d, which show that the surface termination of $\mathrm{CoFe}_{2} \mathrm{O}_{4}$ is oxygen termination under OER conditions. The reaction started between a ligand oxygen on the surface and the adsorbed oxygen species $\left(\mathrm{OH}^{-}\right)$, and the "first" electron transfer step is $\mathrm{O}^{*}+\mathrm{OH}^{-} \rightarrow$ $* \mathrm{OOH}+\mathrm{e}^{-}$. The spin-related OER mechanisms show in Figure 4e. The $\mathrm{FM} \mathrm{CoFe}_{2} \mathrm{O}_{4}$ with FM ligand hole will form oxygen termination with fixed spin direction. The first electron transfer process led to the generation of $\mathrm{O}(\downarrow)^{-}$, that is, the first electron transfer step is spin polarization process to form the triplet state intermediate $\mathrm{O}(\downarrow) \mathrm{O}(\downarrow) \mathrm{H}$ species with a lower barrier (Supplementary Figure 10). Consequently, the triplet state intermediate $\mathrm{O}(\downarrow) \mathrm{O}(\downarrow) \mathrm{H}$ species will prefer to generate the triplet state $\mathrm{O}_{2}$. We also conducted a DFT study on the free energies of OER steps on the (111) surface of $\mathrm{CoFe}_{2} \mathrm{O}_{4}$ with and without spin alignment. The (111) surface is chosen because the TEM investigation found the surface is rich in (111) and there is no remarkbale change on the surface after OER (Supplementary Figure 11). The energy diagram for these two paths to produce triplet oxygen is shown in Figure 4f. The active sites with spin alignment are more thermodynamically favourable to OER, if they associate with ferromagnetic ligand holes, ${ }^{58}$ and the overpotential of producing triplet oxygen is reduced by $390 \mathrm{mV}$ compared to that without aligning spin. The coordinated inter-atomic aligned spin on active sites plays an important role in optimizing the spin-dependent reaction coordinates. 


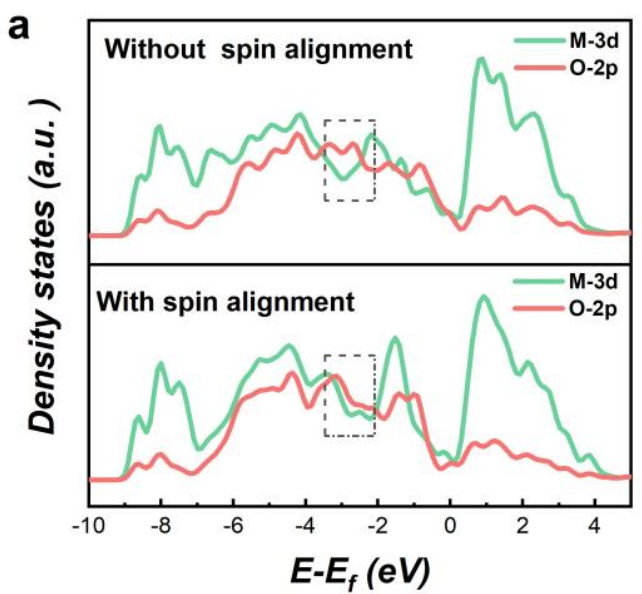

C
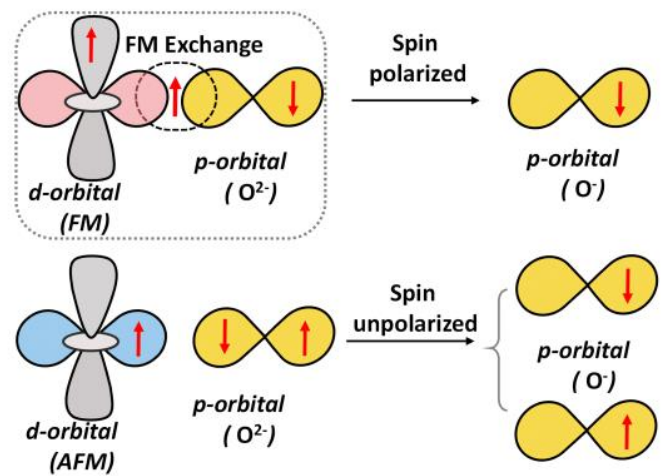

e
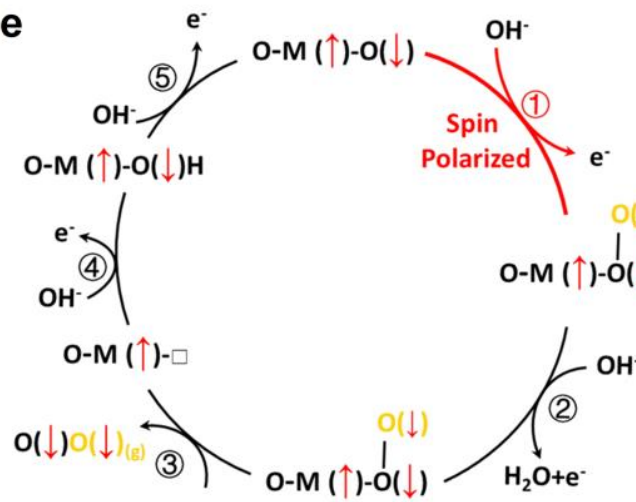

b
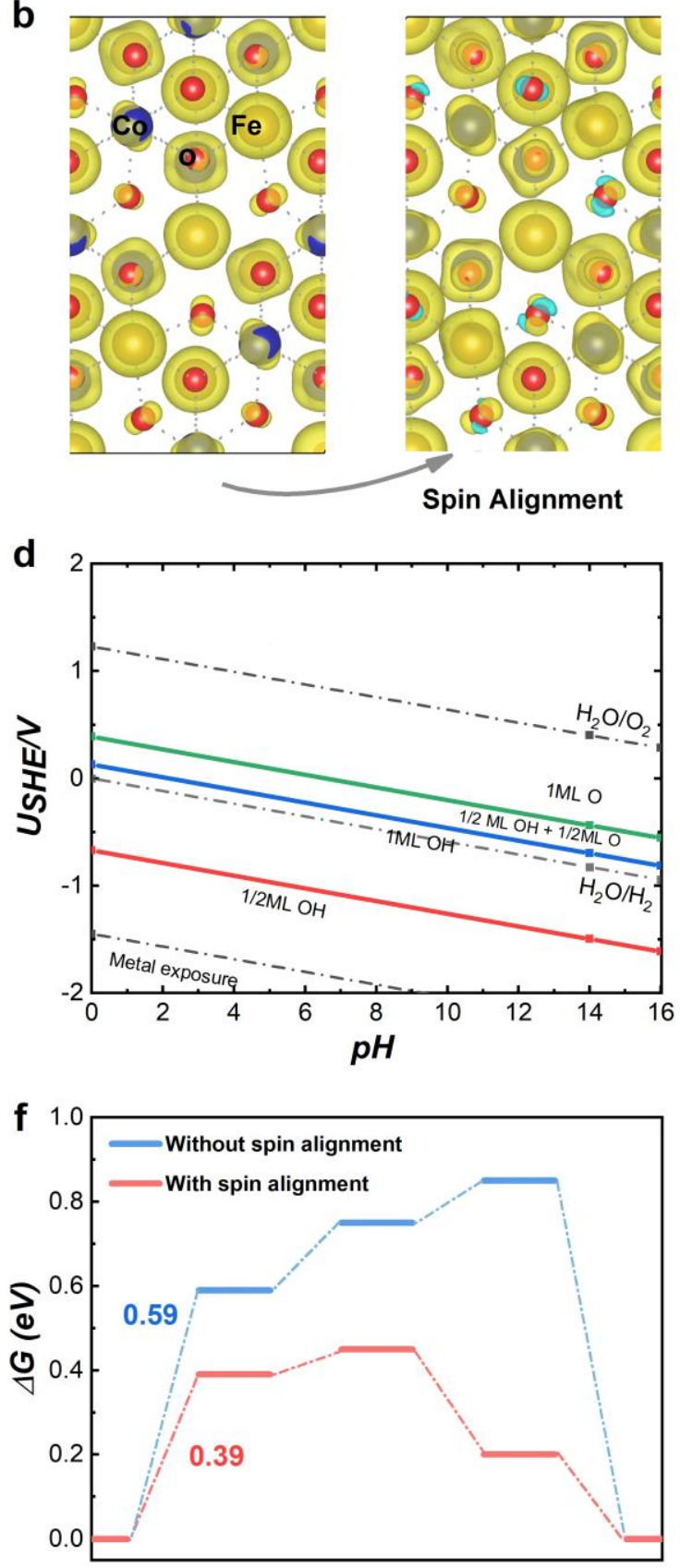

Reaction coordinates

Figure 4 | Spin-polarized OER. (a) The projected density of states (PDOS) of CoFe2O4 without and with spin alignment. (b) The spin density for $\mathrm{CoFe}_{2} \mathrm{O}_{4}$ with and without spin alignment. (c) Schematic of spin-exchange mechanism for OER. The first electron transfer step is promoted by spin polarization through the FM exchange (QSEI), which gives smaller electronic repulsions and makes the adsorbed $\mathrm{O}$ species have a fixed spin direction. (d) The calculated Pourbaix diagram of the (111) surface of $\mathrm{CoFe}_{2} \mathrm{O}_{4}$. (e) The spin polarization 
mechanisms in OER with starting from the step of $\mathrm{O}^{*}+\mathrm{OH}^{-} \rightarrow * \mathrm{OOH}+\mathrm{e}^{-}$step. (f) The free energy diagram of OER at $1.23 \mathrm{~V}$ (vs. RHE) with and without the spin alignment on the (111) surface of $\mathrm{CoFe}_{2} \mathrm{O}_{4}$ toward triplet oxygen production.

It is worth noting that OER requires the generation of paramagnetic $\mathrm{O}_{2}$ molecules starting from diamagnetic species $\left(\mathrm{OH}^{-}\right.$and $\left.\mathrm{H}_{2} \mathrm{O}\right)$. For a reaction involving non-magnetic molecules only, not impact significantly on the reaction kinetics. We investigated the methanol oxidation reaction (MOR) and ethylene glycol oxidation reaction (EGOR) on $\mathrm{CoFe}_{2} \mathrm{O}_{4}$ under the magnetic field. Here, changes in FM catalyst conductivity caused by magnetic fields can be ignored because that $\mathrm{AB}$ mediator is also mixed with $\mathrm{CoFe}_{2} \mathrm{O}_{4}$ for their application as the electrode, which dominant the electron conduction. Figure 5 reveals that there is no remarkable difference in these reactions under the magnetic field. This is because the reactants, intermediates, and the products in these reactions are diamagnetic and there is no spin-selected electron transfer between the active metal site and the adsorbed reaction species.
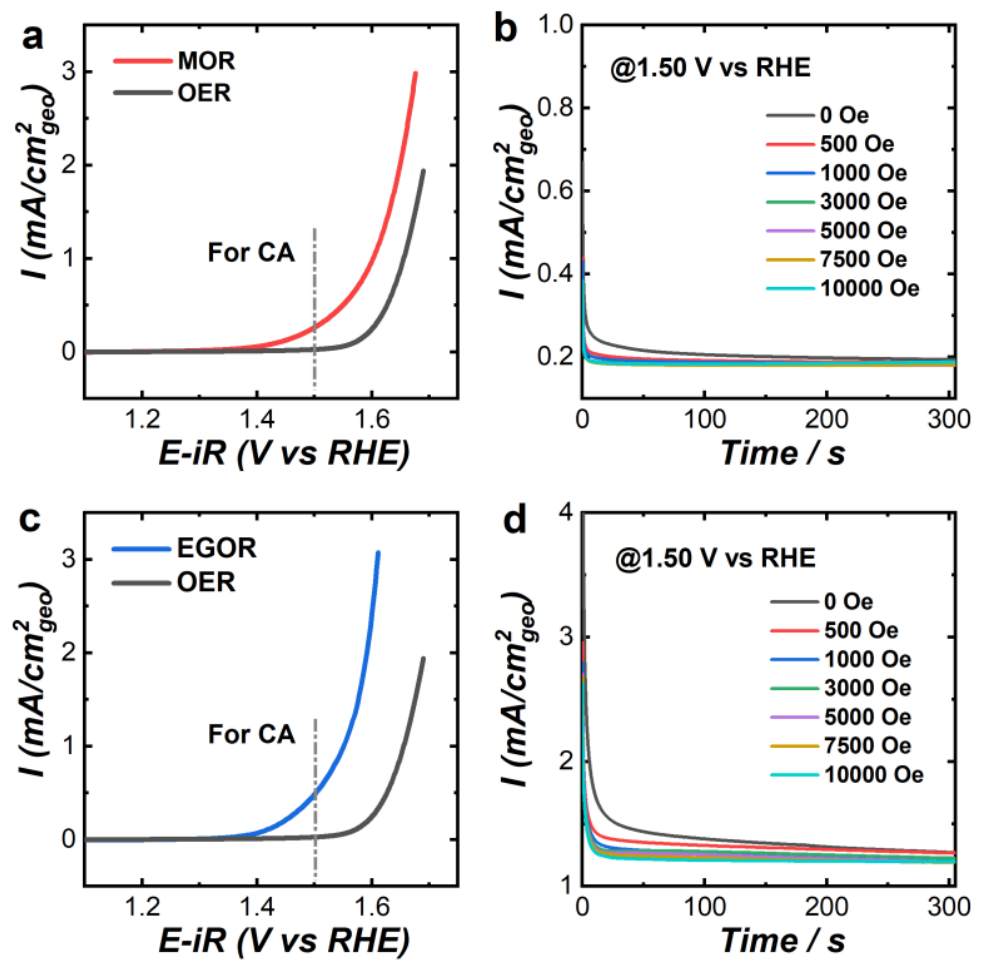

Figure 5 | No influence on MOR and EGOR. (a) Linear sweep voltammetry (LSV) curves of 

$\mathrm{V}$ (versus RHE). (c) LSV curves of EGOR at a scan rate of $10 \mathrm{mV} / \mathrm{s}$ in $1 \mathrm{M} \mathrm{KOH}$ in the presence of $1 \mathrm{ml}$ ethylene glycol. (d) The CA curves of EGOR at $1.50 \mathrm{~V}$ vs. RHE.

\section{The effect of gradient magnetic field, remanence, and demagnetization}

It is known that for ferromagnetic materials, the magnetic moment pertains to the spin. The more ordered its magnetic moment is, the higher the degree of positive spin polarization is. As observed in the initial magnetization curve of $\mathrm{CoFe}_{2} \mathrm{O}_{4}$ (Figure 6a), its magnetic moments become more orderly as the magnetic field increases, before reaching the saturation field. We then investigated the effect of the gradient magnetic field on OER activity. We carried out a series of CA measurements under the different magnetic field strength at a constant potential of $1.66,1.66$, and $1.56 \mathrm{~V}$ (versus $\mathrm{RHE}$ ) for $\mathrm{CoFe}_{2} \mathrm{O}_{4}, \mathrm{Co}_{3} \mathrm{O}_{4}$, and $\mathrm{IrO}_{2}$ respectively (Figure 6b). It can be seen that the current density of the ferromagnetic catalyst $\mathrm{CoFe}_{2} \mathrm{O}_{4}$ increases with the increase of the magnetic field strength. For non-ferromagnetic $\mathrm{Co}_{3} \mathrm{O}_{4}$ and $\mathrm{IrO}_{2}$, there is almost no change when changing the field strength. The increment of the current density is summarized in Figure 6c. The increase of spatial spin polarization related to the degree of magnetization shows a positive correlation with the enhancement of the OER for ferromagnetic $\mathrm{CoFe}_{2} \mathrm{O}_{4}$. We also measured the LSV curves of all oxides before and after the CA experiment. As seen in Supplementary Figure 12, the OER performance ferromagnetic $\mathrm{CoFe}_{2} \mathrm{O}_{4}$ can be further improved after the CA test under magnetic field, but not for non-ferromagnetic $\mathrm{Co}_{3} \mathrm{O}_{4}$ and $\mathrm{IrO}_{2}$. An interesting finding is that the OER performance of $\mathrm{CoFe}_{2} \mathrm{O}_{4}$ remains even after the magnetic field is removed (Figure 6d). This is because the magnetic moment is still aligned in magnetized $\mathrm{CoFe}_{2} \mathrm{O}_{4}$ (Figure 6e) after removing the magnetic field, which persists as the spin polarizer to create spin polarization. This is an important fact to make clear that the enhancement is due to the indirect (strong) QSEI, and not due to weak direct spin-spin interactions from the external field, a typically conceptual error. More interestingly, when the magnetized $\mathrm{CoFe}_{2} \mathrm{O}_{4}$ was demagnetized using an oscillating magnetic field (Figure 6g), the OER performance of $\mathrm{CoFe}_{2} \mathrm{O}_{4}$ 
reverted to the initial value before the field was applied. The Tafel slope of $\mathrm{CoFe}_{2} \mathrm{O}_{4}$ is back to $120 \mathrm{mV} \mathrm{dec}^{-1}$, indicating the first electron transfer of the adsorbed $\mathrm{OH}^{-}$is again the $\mathrm{RDS}$, same 341 as the status without the magnetic field. Based on the above results, we can confirm that the spin polarization facilitated OER is reversible and adjustable.
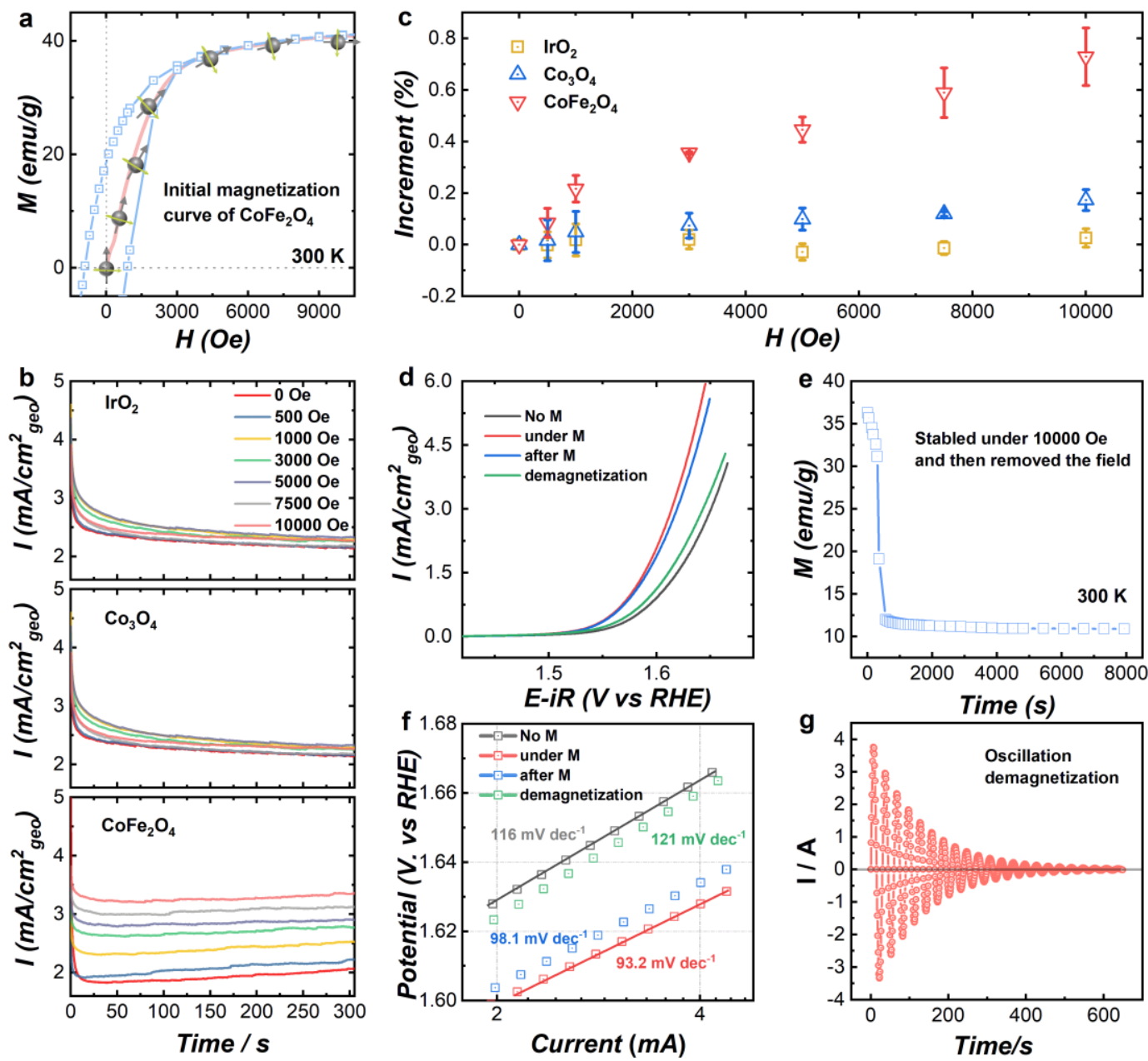

Figure 6 | The effect of gradient magnetic field, remanence, and demagnetization. (a)

Initial magnetization curve of $\mathrm{CoFe}_{2} \mathrm{O}_{4}$. (b) $\mathrm{CA}$ test in $1 \mathrm{M} \mathrm{KOH}$ under the different magnetic field strength $(0,500,1000,3000,5000,7500$, and $10000 \mathrm{Oe})$ at a constant potential of $1.66 \mathrm{~V}$ versus RHE for $\mathrm{CoFe}_{2} \mathrm{O}_{4}, \mathrm{Co}_{3} \mathrm{O}_{4}$, and $1.56 \mathrm{~V}$ versus $\mathrm{RHE}$ for $\mathrm{IrO}_{2}$. (c) The increment of the current density under different magnetic field strength. It was calculated by the following equation: Increment $(\%)=\left(\mathrm{j}_{\mathrm{M}}-\mathrm{j}_{\mathrm{M}=0}\right) / \mathrm{j}_{\mathrm{M}=0} ; \mathrm{j}_{\mathrm{M}}$ is the chronopotentiometry current density values 350 obtained under the applied magnetic fields $(0,500,1000,3000,5000,7500$, and $10000 \mathrm{Oe})$.

351 The error bar represents three independent tests. (d) LSV curves of $\mathrm{CoFe}_{2} \mathrm{O}_{4}$ at a scan rate of 
$10 \mathrm{mV} / \mathrm{s}$ in $\mathrm{O}_{2}$-saturated $1 \mathrm{M} \mathrm{KOH}$ with and without a constant magnetic field (10000 Oe), after the magnetic field removed (after M), and after demagnetization. The corresponding Tafel plots are shown in (f). (e) The magnetization of $\mathrm{CoFe}_{2} \mathrm{O}_{4}$ after removing a constant magnetic field of 10000 Oe. (g) The curve of demagnetization for $\mathrm{CoFe}_{2} \mathrm{O}_{4}$.

\section{Conclusion}

In summary, we have found that ferromagnetic $\mathrm{CoFe}_{2} \mathrm{O}_{4}$ as the spin polarizer facilitates the spin polarization under a constant magnetic field, because macroscopic ferromagnetic QSEI promote the OER activity. The increase of spatial spin polarization shows a positive correlation with the enhancement of spin-transport (selection) during OER. We have found that the Tafel slope of overall ferromagnetic $\mathrm{CoFe}_{2} \mathrm{O}_{4}$ switched from $\sim 120$ to $\sim 90 \mathrm{mV} \cdot \mathrm{dec}^{-1}$ after applying a magnetic field. It indicates the change of the RDS of OER reaction under an external magnetic field, i.e., the first electron transfer step is no longer the RDS. The spin-polarized electron exchange between the ferromagnetic $\mathrm{CoFe}_{2} \mathrm{O}_{4}$ and the adsorbed oxygen species (reactants) for the first electron transfer is ferromagnetic exchange under the principle of spin angular momentum conservation, which leads to faster reaction kinetics for the first electron transfer step. In contrast, such a phenomenon was not observed on non-ferromagnetic catalysts. The findings imply that the conservation of the total spin on the active sites during OER is an important concept, which applies quantum spin-exchange interactions to optimize reaction kinetics. The kinetic improvements maintain after the removal of the external magnetic field, and our work proves that a new path is available in the development of clean and more efficient catalysts for hydrogen economy.

\section{Methods}

Oxides synthesis. Spinel $\mathrm{CoFe}_{2} \mathrm{O}_{4}$ oxides were synthesized by a modified conventional solidstate chemistry method as described elsewhere ${ }^{45}$ with $\mathrm{Fe}\left(\mathrm{NO}_{3}\right)_{2}$ and $\mathrm{Co}\left(\mathrm{NO}_{3}\right)_{2}$ as precursors. 9 
mmol mixture of $\mathrm{Fe}\left(\mathrm{NO}_{3}\right)_{3} \cdot 9 \mathrm{H}_{2} \mathrm{O}$ (Alfa Aesar) and $\mathrm{Co}\left(\mathrm{NO}_{3}\right)_{2} \cdot 6 \mathrm{H}_{2} \mathrm{O}$ (Sigma Aldrich) was dissolved in $15 \mathrm{~mL}$ of DI water, followed by stirring and vaporizing in oven at $80{ }^{\circ} \mathrm{C}$. The resulting slurry was calcinated at $250{ }^{\circ} \mathrm{C}$ for 2 hours in the air to decompose nitrous completely. After grinding, the black oxide powders underwent calcination in air at $400{ }^{\circ} \mathrm{C}$ for 8 hours. $\mathrm{Co}_{3} \mathrm{O}_{4}$ oxides were synthesized by the same method.

Electrochemical characterization. The OER tests were operated in a three-electrode cell with a working electrode (WE) of glassy carbon flake $(10 \mathrm{~mm} \times 20 \mathrm{~mm} \times 0.5 \mathrm{~mm}$; Effective electrode area: $1.0 \mathrm{~cm}^{2}$ ), a counter electrode of platinum foil, and a $\mathrm{Hg} / \mathrm{HgO}$ reference electrode (RE) (filled with $1 \mathrm{M} \mathrm{KOH}$ solution). The catalysts electrode was fabricated by the recipe drop-castes method which was reported in elsewhere ${ }^{59}$. The catalysts were mixed with acetylene black (AB) at a mass ration of $5: 1$, then were dispersed in isopropanol/water $(\mathrm{v} / \mathrm{v}=1: 4)$ solvent followed by the addition of $\mathrm{Na}^{+}$-exchanged Nafion as the binder. The mixtures were ultrasonicated for 30 min to reach homogeneous ink. The concentration of oxides in ink is $5 \mathrm{mg} / \mathrm{ml}$, and $\mathrm{AB}$ is 1 $\mathrm{mg} / \mathrm{ml}$. Before drop-casting, the glassy carbon electrodes were polished to a mirror finish with $\alpha-\mathrm{Al}_{2} \mathrm{O}_{3}(50 \mathrm{~nm})$ and washed by IPA and water to clean up completely. Finally, the as-prepared ink (100 ul) was dropped onto glassy carbon electrodes to reach a loading mass of $500 \mu \mathrm{g}_{\mathrm{ox}} \mathrm{cm}^{-}$ ${ }^{2}$ and the electrodes were dried overnight at room temperature. Cyclic voltammograms (CVs), linear sweep voltammetry (LSV) and chronoamperometry (CA) were performed in $\mathrm{O}_{2}$ saturated $1 \mathrm{M} \mathrm{KOH}$ by using Bio-logic SP 150 potentiostat. All potentials were converted to RHE scale according to the following equation: $\mathrm{RHE}=\mathrm{Hg} / \mathrm{HgO}+0.098$ with iR correction . The tests of methanol oxidation reaction (MOR) and ethylene glycol oxidation reaction (EGOR) on CFO electrodes are similar to the OER test. The difference is that the MOR and EGOR were studied in $1 \mathrm{M} \mathrm{KOH} 100 \mathrm{ml}$ electrolyte in the presence of $1 \mathrm{ml}$ methanol and $1 \mathrm{ml}$ ethylene glycol, respectively ${ }^{60}$.

Materials characterization. The X-ray diffraction (XRD) of oxides were carried on Bruker D8 diffractometer at a scanning rate of $2^{\circ} \mathrm{min}^{-1}$, under $\mathrm{Cu}-\mathrm{K}_{\alpha}$ radiation $(\lambda=1.5418 \AA)$. DC magnetization measurements were performed on a Superconducting Quantum Design (SQUID) 
magnetometer (MPMS-XL). The SQUID measurements of the magnetization of samples as a function of the magnetic field were carried out at $300 \mathrm{~K}$ in fields between $-5 \mathrm{~T}$ and $+5 \mathrm{~T}$. The high-resolution transmission electron microscopy (HRTEM) was carried JEOL JEM- 2100 plus microscope at 200KV. The STEM results presented here were obtained using the 200kV JEOL ARM electron microscope equipped (JEOL, Tokyo, Japan) with double aberration correctors, a dual-energy-loss spectrometer and a cold field emission source. The atomic-resolved STEM images were collected with a condense aperture of $28 \mathrm{mrad}$ and a collection angle of $90-370$ mrad for HAADF and $11-23$ mrad for ABF images. The XPS measurements were performed using PHI-5400 equipment with $\mathrm{Al} \mathrm{K} \alpha$ beam source $(250 \mathrm{~W})$ and a position-sensitive detector (PSD) was used to determine the surface composition of the materials. The Fourier transform infrared spectroscopy-Raman spectroscopy was carried with a confocal Raman microscope (Horiba HR Evolution), equipped with a diode laser emitting at $532 \mathrm{~nm}$. The nominal laser power was filtered down to $1 \mathrm{~mW}$ to avoid sample overheating. Spectra were recorded with the accumulation time of $60 \mathrm{~s}$.

DFT studies. All the density functional theory (DFT) calculations were performed by Vienna Ab-initio Simulation Package ${ }^{61,62}$ (VASP), employing the Projected Augmented Wave ${ }^{63}$ (PAW) method. The revised Perdew-Burke-Ernzerhof (RPBE) functional was used to describe the exchange and correlation effects. ${ }^{64-66}$ The GGA + U calculations are performed using the model proposed by Dudarev et al. ${ }^{67}$, with the $\mathrm{U}_{\text {eff }}\left(\mathrm{U}_{\text {eff }}=\right.$ Coulomb $\mathrm{U}-$ exchange $\left.\mathrm{J}\right)$ values of $3.3 \mathrm{eV}$ and $4 \mathrm{eV}$ for $\mathrm{Co}$ and $\mathrm{Fe}$, respectively. For all the geometry optimizations, the cutoff energy was set to be $500 \mathrm{eV}$. A $3 \times 3 \times 1$ Monkhorst-Pack grids ${ }^{68}$ was used to carry out the surface calculations on the (111) surface of $\mathrm{CoFe}_{2} \mathrm{O}_{4}$. At least $20 \AA$ vacuum layer was applied in z-direction of the slab models, preventing the vertical interactions between slabs.

In alkaline conditions, OER could occur in the following four elementary steps:

$\mathrm{OH}^{-}+* \rightarrow * \mathrm{OH}+\mathrm{e}^{-}$

$* \mathrm{OH}+\mathrm{OH}^{-} \rightarrow * \mathrm{O}+\mathrm{H}_{2} \mathrm{O}+\mathrm{e}^{-}$

$* \mathrm{O}+\mathrm{OH}^{-} \rightarrow * \mathrm{OOH}+\mathrm{e}^{-}$ 
439 where * denotes the active sites on the catalyst surface. Based on the above mechanism, the 440 free energy of three intermediate states, ${ }^{*} \mathrm{OH},{ }^{*} \mathrm{O}$, and ${ }^{*} \mathrm{OOH}$, are important to identify a given material's OER activity. The computational hydrogen electrode (CHE) model ${ }^{69}$ was used to calculate the free energies of OER, based on which the free energy of an adsorbed species is defined as

$\Delta \mathrm{G}_{\mathrm{ads}}=\Delta \mathrm{E}_{\mathrm{ads}}+\Delta \mathrm{E}_{\mathrm{ZPE}}-\mathrm{T} \Delta \mathrm{S}_{\mathrm{ads}}$

where $\Delta E_{a d s}$ is the electronic adsorption energy, $\Delta E_{Z P E}$ is the zero point energy difference between adsorbed and gaseous species, and $T \Delta S_{a d s}$ is the corresponding entropy difference between these two states. The electronic binding energy is referenced as $1 / 2 \mathrm{H}_{2}$ for each $\mathrm{H}$ atom, and $\left(\mathrm{H}_{2} \mathrm{O}-\mathrm{H}_{2}\right)$ for each $\mathrm{O}$ atom, plus the energy of the clean slab. The corrections of zero point energy and entropy of the OER intermediates can be found in the Supplementary Table 2.

\section{References}

1. Young, J.L. et al. Direct solar-to-hydrogen conversion via inverted metamorphic multi-junction

2. Aurbach, D., McCloskey, B.D., Nazar, L.F. \& Bruce, P.G. Advances in understanding mechanisms underpinning lithium-air batteries. Nature Energy 1, 16128 (2016).

3. Duan, C. et al. Highly durable, coking and sulfur tolerant, fuel-flexible protonic ceramic fuel

4. Cheng, W. et al. Lattice-strained metal-organic-framework arrays for bifunctional oxygen

5. Koshikawa, H. et al. Single Nanometer-Sized NiFe-Layered Double Hydroxides as Anode Catalyst electrocatalysis. Nature Energy 4, 115-122 (2019).

in Anion Exchange Membrane Water Electrolysis Cell with Energy Conversion Efficiency of 74.7\% at $1.0 \mathrm{~A} / \mathrm{cm}^{2}$. ACS Catalysis 10, 1886-1893 (2020).

6. Stoerzinger, K.A., Qiao, L., Biegalski, M.D. \& Shao-Horn, Y. Orientation-Dependent Oxygen 7. Hong, W.T. et al. Toward the rational design of non-precious transition metal oxides for oxygen electrocatalysis. Energy \& Environmental Science 8, 1404-1427 (2015).

8. Calle-Vallejo, F. et al. Number of outer electrons as descriptor for adsorption processes on transition metals and their oxides. Chemical Science 4, 1245-1249 (2013).

9. Man, I.C. et al. Universality in Oxygen Evolution Electrocatalysis on Oxide Surfaces.

10. Suntivich, J., May, K.J., Gasteiger, H.A., Goodenough, J.B. \& Shao-Horn, Y. A perovskite oxide optimized for oxygen evolution catalysis from molecular orbital principles. Science $\mathbf{3 3 4}, \mathbf{1 3 8 3 -}$ 
1385 (2011).

11. Chao, W. et al. Cations in Octahedral Sites: A Descriptor for Oxygen Electrocatalysis on Transition-Metal Spinels. Adv. Mater. 29, 1606800 (2017).

12. Suntivich, J. et al. Design principles for oxygen-reduction activity on perovskite oxide catalysts for fuel cells and metal-air batteries. Nat Chem 3, 546-550 (2011).

13. Tingbin Lim, J.W.H.N., and Jose Gracia Layered Antiferromagnetic Ordering in the Most Active Perovskite Catalysts for the Oxygen Evolution Reaction. ChemCatChem 8, 2968-2974 (2016).

14. Ryan Sharpe, J.M., Tingbin Lim, Yunzhe Jiao, J. W. Niemantsverdriet, Victor Polo, and Jose Gracia Orbital Physics of Perovskites for the Oxygen Evolution Reaction. Top. Catal. 61, 267275 (2018).

15. Sharpe, R., Lim, T., Jiao, Y., Niemantsverdriet, J.W.H. \& Gracia, J. Oxygen Evolution Reaction on Perovskite Electrocatalysts with Localized Spins and Orbital Rotation Symmetry. ChemCatChem 8, 3762-3768 (2016).

16. Gracia, J. et al. Analysis of the Magnetic Entropy in Oxygen Reduction Reactions Catalysed by Manganite Perovskites. ChemCatChem 9, 3358-3363 (2017).

17. Chretien, S. \& Metiu, H. O $\mathrm{O}_{2}$ evolution on a clean partially reduced rutile $\mathrm{TiO}_{2}(110)$ surface and on the same surface precovered with Au1 and Au2: the importance of spin conservation. $J$. Chem. Phys. 129, 074705 (2008).

18. Torun, E., Fang, C.M., de Wijs, G.A. \& de Groot, R.A. Role of Magnetism in Catalysis: $\mathrm{RuO}_{2}(110)$ Surface. J. Phys. Chem. C 117, 6353-6357 (2013).

19. Nazmutdinov, R.R., Santos, E. \& Schmickler, W. Spin effects in oxygen electrocatalysis: A discussion. Electrochem. Commun. 33, 14-17 (2013).

20. A. V. Ramos, T.S.S., G. X. Miao, M.-J. Guittet, J.-B. Moussy, and J. S. Moodera Influence of oxidation on the spin-filtering properties of $\mathrm{CoFe}_{2} \mathrm{O}_{4}$ and the resultant spin polarization. Phys. Rev. B 78, 180402 (2008).

21. Zhi-Min Liao, Y.-D.L., Jun Xu, Jing-Min Zhang, Ke Xia, and Da-Peng Yu Spin-Filter Effect in Magnetite Nanowire. Nano Lett. 6, 1087-1091 (2006).

22. Gracia, J. Itinerant Spins and Bond Lengths in Oxide Electrocatalysts for Oxygen Evolution and Reduction Reactions. J. Phys. Chem. C 123, $9967-9972$ (2019).

23. Gracia, J., Sharpe, R. \& Munarriz, J. Principles determining the activity of magnetic oxides for electron transfer reactions. J. Catal. 361, 331-338 (2018).

24. Gracia, J. Spin dependent interactions catalyse the oxygen electrochemistry. Phys. Chem. Chem. Phys. 19, 20451-20456 (2017).

25. Mtangi, W. et al. Control of Electrons' Spin Eliminates Hydrogen Peroxide Formation During Water Splitting. J. Am. Chem. Soc. 139, 2794-2798 (2017).

26. Zhang, W., Banerjee-Ghosh, K., Tassinari, F. \& Naaman, R. Enhanced Electrochemical Water Splitting with Chiral Molecule-Coated $\mathrm{Fe}_{3} \mathrm{O}_{4}$ Nanoparticles. ACS Energy Letters 3, 2308-2313 (2018).

27. Mondal, P.C., Fontanesi, C., Waldeck, D.H. \& Naaman, R. Spin-Dependent Transport through Chiral Molecules Studied by Spin-Dependent Electrochemistry. Acc. Chem. Res. 49, 2560-2568 (2016).

28. Jiao, Y., Sharpe, R., Lim, T., Niemantsverdriet, J.W.H. \& Gracia, J. Photosystem II Acts as a SpinControlled Electron Gate during Oxygen Formation and Evolution. J. Am. Chem. Soc. 139, 
16604-16608 (2017).

29. Lüders, U. et al. Spin filtering through ferrimagnetic $\mathrm{NiFe}_{2} \mathrm{O}_{4}$ tunnel barriers. Appl. Phys. Lett. 88, 082505 (2006).

30. Garcés-Pineda, F.A., Blasco-Ahicart, M., Nieto-Castro, D., López, N. \& Galán-Mascarós, J.R. Direct magnetic enhancement of electrocatalytic water oxidation in alkaline media. Nature Energy 4, 519-525 (2019).

31. Yiyun Yang, K.M.G., Henry S. White, and Shaowei Chen Magnetoelectrochemistry of Nitrothiophenolate-Functionalized Gold Nanoparticles. Langmuir 19, 9446-9449 (2003).

32. Monzon, L.M.A. \& Coey, J.M.D. Magnetic fields in electrochemistry: The Kelvin force. A minireview. Electrochem. Commun. 42, 42-45 (2014).

33. Monzon, L.M.A. \& Coey, J.M.D. Magnetic fields in electrochemistry: The Lorentz force. A minireview. Electrochem. Commun. 42, 38-41 (2014).

34. Olivier Devos, O.A., Jean-Paul Chopart, and Alain Olivier Is There a Magnetic Field Effect on Electrochemical Kinetics? J. Phys. Chem. A 104, 1544-1548 (2000).

35. Gatard, V., Deseure, J. \& Chatenet, M. Use of magnetic fields in electrochemistry: a selected review. Current Opinion in Electrochemistry 23, 96-105 (2020).

36. Monzon, L.M.A., Nair, V., Reilly, B. \& Coey, J.M.D. Magnetically-Induced Flow during Electropolishing. J. Electrochem. Soc. 165, E679-E684 (2018).

37. Lee du, H., Choi, C.H., Choi, T.H., Sung, B.J. \& Kang, H. Asymmetric Transport Mechanisms of Hydronium and Hydroxide Ions in Amorphous Solid Water: Hydroxide Goes Brownian while Hydronium Hops. J.Phys. Chem. Lett. 5, 2568-2572 (2014).

38. Chappert, C., Fert, A. \& Van Dau, F.N. The emergence of spin electronics in data storage. Nat. Mater. 6, 813-823 (2007).

39. Binasch, G., Grunberg, P., Saurenbach, F. \& Zinn, W. Enhanced magnetoresistance in layered magnetic structures with antiferromagnetic interlayer exchange. Phys Rev B Condens Matter 39, 4828-4830 (1989).

40. Zhou, B.H. \& Rinehart, J.D. A Size Threshold for Enhanced Magnetoresistance in Colloidally Prepared $\mathrm{CoFe}_{2} \mathrm{O}_{4}$ Nanoparticle Solids. ACS Cent Sci 4, 1222-1227 (2018).

41. Jun Chen, X., Soong Ju Oh, JamesM. Kikkawa, Cherie R. Kagan, and Christopher B. Murray Bistable Magnetoresistance Switching in Exchange-Coupled $\mathrm{CoFe}_{2} \mathrm{O}_{4}-\mathrm{Fe}_{3} \mathrm{O}_{4}$ Binary Nanocrystal Superlattices by Self-Assembly and Thermal Annealing. ACS Nano. 7, 1478-1486 (2013).

42. Gul, I.H. \& Maqsood, A. Structural, magnetic and electrical properties of cobalt ferrites prepared by the sol-gel route. J. Alloys Compd. 465, 227-231 (2008).

43. Xu, Z.J. From Two-Phase to Three-Phase: The New Electrochemical Interface by Oxide Electrocatalysts. Nanomicro Lett 10, 8 (2018).

44. Xu, Z.J. Transition metal oxides for water oxidation: All about oxyhydroxides? Science China Materials 63, 3-7 (2019).

45. Wu, T. et al. Iron-facilitated dynamic active-site generation on spinel $\mathrm{CoAl}_{2} \mathrm{O}_{4}$ with selftermination of surface reconstruction for water oxidation. Nature Catalysis 2, 763-772 (2019).

46. Chen, Y. et al. Exceptionally active iridium evolved from a pseudo-cubic perovskite for oxygen evolution in acid. Nat. Commun. 10, 572-582 (2019).

47. El Arrassi, A. et al. Intrinsic Activity of Oxygen Evolution Catalysts Probed at Single $\mathrm{CoFe}_{2} \mathrm{O}_{4}$ Nanoparticles. J. Am. Chem. Soc. 141, 9197-9201 (2019). 
48. Chandramohan, P., Srinivasan, M.P., Velmurugan, S. \& Narasimhan, S.V. Cation distribution and particle size effect on Raman spectrum of $\mathrm{CoFe}_{2} \mathrm{O}_{4}$. J. Solid State Chem. 184, 89-96 (2011).

49. Zhou, Z. et al. Electronic structure studies of the spinel $\mathrm{CoFe}_{2} \mathrm{O}_{4}$ by X-ray photoelectron spectroscopy. Appl. Surf. Sci. 254, 6972-6975 (2008).

50. Fang, Y.-H. \& Liu, Z.-P. Tafel Kinetics of Electrocatalytic Reactions: From Experiment to FirstPrinciples. ACS Catalysis 4, 4364-4376 (2014).

51. Sun, S. et al. Switch of the Rate-Determining Step of Water Oxidation by Spin-Selected Electron Transfer in Spinel Oxides. Chem. Mater. 31, 8106-8111 (2019).

52. Duan, Y. et al. Revealing the Impact of Electrolyte Composition for Co-Based Water Oxidation Catalysts by the Study of Reaction Kinetics Parameters. ACS Catalysis 10, 4160-4170 (2020).

53. Craig, M.J. et al. Universal scaling relations for the rational design of molecular water oxidation catalysts with near-zero overpotential. Nature Communications 10 (2019).

54. García-Melchor, M., Vilella, L., López, N. \& Vojvodic, A. Computationally Probing the Performance of Hybrid, Heterogeneous, and Homogeneous Iridium-Based Catalysts for Water Oxidation. ChemCatChem 8, 1792-1798 (2016).

55. Wei, C. et al. Cations in Octahedral Sites: A Descriptor for Oxygen Electrocatalysis on TransitionMetal Spinels. Adv. Mater. 29, 1606800 (2017).

56. J. Teillet, F.B.a.R.K. Magnetic structure of CoFe2O4. J. Magn. Magn. Mater. 123, 93-96 (1993).

57. M. Guiilot, j.O., and A. Marchand High magnetic field magnetization study in cadmium-cobalt ferrite single crystals. Condens. Matter Phys. 71, 193-197 (1988).

58. Zhang, L., Cheruvathur, A., Biz, C., Fianchini, M. \& Gracia, J. Ferromagnetic ligand holes in cobalt perovskite electrocatalysts as an essential factor for high activity towards oxygen evolution. Phys. Chem. Chem. Phys. 21, 2977-2983 (2019).

59. Suntivich, J., Gasteiger, H.A., Yabuuchi, N. \& Shao-Horn, Y. Electrocatalytic Measurement Methodology of Oxide Catalysts Using a Thin-Film Rotating Disk Electrode. J. Electrochem. Soc. 157, B1263-B1268 (2010).

60. Sun, S., Zhou, Y., Hu, B., Zhang, Q. \& Xu, Z.J. Ethylene Glycol and Ethanol Oxidation on Spinel $\mathrm{Ni}-\mathrm{Co}$ Oxides in Alkaline. J. Electrochem. Soc. 163, H99-H104 (2015).

61. Kresse, G. \& Furthmüller, J. Efficient iterative schemes for ab initio total-energy calculations using a plane-wave basis set. Phys. Rev. B 54, 11169-11186 (1996).

62. Kresse, G. \& Hafner, J. Ab initio molecular dynamics for liquid metals. Phys. Rev. B 49, 1425114269 (1994).

63. Blöchl, P.E. Projector augmented-wave method. Phys. Rev. B 50, 17953-17979 (1994).

64. Perdew, J.P., Burke, K. \& Ernzerhof, M. Generalized Gradient Approximation Made Simple. Phys. Rev. Lett. 77, 3865-3868 (1996).

65. Zhang, Y. \& Yang, W. Comment on "Generalized Gradient Approximation Made Simple". Phys. Rev. Lett. 80, 890-890 (1998).

66. Hammer, B., Hansen, L.B. \& Nørskov, J.K. Improved adsorption energetics within densityfunctional theory using revised Perdew-Burke-Ernzerhof functionals. Phys. Rev. B 59, 74137421 (1999).

67. Dudarev, S., Botton, G., Savrasov, S., Humphreys, C. \& Sutton, A. Electron-energy-loss spectra and the structural stability of nickel oxide: An LSDA+ U study. Phys. Rev. B 57, 1505 (1998).

68. Monkhorst, H.J. \& Pack, J.D. Special points for Brillouin-zone integrations. Phys. Rev. B 13, 5188 
602

603

604

605

606

607

608

609

610

611

612

613

614

615

616

617

618

619

620

621

622

623

624

625

626

627

628

629

630

631

(1976).

69. Nørskov, J.K. et al. Origin of the overpotential for oxygen reduction at a fuel-cell cathode. The Journal of Physical Chemistry B 108, 17886-17892 (2004).

\section{Acknowledgements}

The authors thank the support from the National Nature Science Foundation of China (Grant No. 11274370, and 51471185) and the National National Key R\&D Program of China (Grant No. 2016YFJC020013 and 2018FYA0305800). Authors in Singapore thank the support from the Singapore Ministry of Education Tier 2 Grants (MOE2017-T2-1-009 and MOE2018-T2-2027) and the Singapore National Research Foundation under its Campus for Research Excellence and Technological Enterprise (CREATE) programme. Authors appreciate the Facility for Analysis, Characterisation, Testing and Simulation (FACTS) at Nanyang Technological University for materials characterizations. This work was supported by the National Research Foundation (NRF), Prime Minister's Office, Singapore under its Campus for Research Excellence and Technological Enterprise (CREATE) programme through the eCO2EP project operated by the Cambridge Centre for Advanced Research and Education in Singapore (CARES) and the Berkeley Educational Alliance for Research in Singapore (CARES).

\section{Author contributions}

X.R., T.W. and Y.S. contribute equally to this work. Z.X., X.R., T.W. conceived the original concept and designed the experiments. T.W. prepared the materials. X. R. performed most characterizations and analysis. G.X. and X.R. performed the Raman spectroscopy measurement. H.Y., X. R. and L.Y. carried out magnetic property measurements and analysis. Y.S. contributed the DFT calculations and analysis. T.W., X.R., and Z.X. contributed the mechanism analysis. J.G. contributed the explanation of QSEI theory. X.R., T.W., Y.S., H.Y., and Z.X wrote the manuscript with the input from all authors. All authors engaged in the analysis of experimental results and manuscript edition.

\section{Data availability statement}


632 The data that support the findings of this study are available from the corresponding author 633 upon reasonable request.

634

635

636 Competing interests

637 The authors declare no competing financial interests.

638

639 Additional information

640 Supplementary information is available for this paper at

641

642 


\section{Supplementary materials}

\section{Spin-polarized Oxygen Evolution Reaction under Magnetic Field}

4 Xiao Ren ${ }^{1,2}$, Tianze $\mathrm{Wu}^{1,2,3}$, Yuanmiao Sun ${ }^{2}$, Yan $\mathrm{Li}^{1}$, Guoyu Xian ${ }^{1}$, Xianhu Liu ${ }^{4}$, Chengmin

5 Shen $^{1}$, Jose Gracia ${ }^{5}$, Hong-Jun Gao ${ }^{1}$, Haitao Yang ${ }^{l *}$, Zhichuan J. Xu ${ }^{2,3,6 *}$

6

$7 \quad{ }^{1}$ Beijing National Laboratory for Condensed Matter Physics and Institute of Physics, Chinese

8 Academy of Science, P.O.Box 603, Beijing, 100190, China;

$9 \quad{ }^{2}$ School of Material Science and Engineering, Nanyang Technological University, 50 Nanyang

10 Avenue, Singapore 639798, Singapore;

$11{ }^{3}$ Solar Fuels Laboratory, Nanyang Technological University, 50 Nanyang Avenue, Singapore

12 639798, Singapore;

$13 \quad{ }^{4}$ Key Laboratory of Advanced Material Processing \& Mold (Zhengzhou University), Ministry

14 of Education, Zhengzhou 450002, China;

$15{ }^{5}$ MagnetoCat SL, General Polavieja 9 3I, 03012 Alicante, Spain;

$16{ }^{6}$ Energy Research Institute @ Nanyang Technological University, 50 Nanyang Avenue,

17 Singapore 639798, Singapore.

18 E-mails: xuzc@ntu.edu.sg; htyang@iphy.ac.cn 
Oxides synthesis. Spinel $\mathrm{CoFe}_{2} \mathrm{O}_{4}$ oxides were synthesized by a modified conventional solid-state chemistry method as described elsewhere ${ }^{1}$ with $\mathrm{Fe}\left(\mathrm{NO}_{3}\right)_{2}$ and $\mathrm{Co}\left(\mathrm{NO}_{3}\right)_{2}$ as precursors. 9 mmol mixture of $\mathrm{Fe}\left(\mathrm{NO}_{3}\right)_{3} \cdot 9 \mathrm{H}_{2} \mathrm{O}$ (Alfa Aesar) and $\mathrm{Co}\left(\mathrm{NO}_{3}\right)_{2} \cdot 6 \mathrm{H}_{2} \mathrm{O}$ (Sigma Aldrich) was dissolved in $15 \mathrm{~mL}$ of DI water, followed by stirring and vaporizing in oven at $80{ }^{\circ} \mathrm{C}$. The resulting slurry was calcinated at $250{ }^{\circ} \mathrm{C}$ for 2 hours in the air to decompose nitrous completely. After grinding, the black oxide powders underwent calcination in air at $400{ }^{\circ} \mathrm{C}$ for 8 hours. $\mathrm{Co}_{3} \mathrm{O}_{4}$ oxides were synthesized by the same method.

Electrochemical characterization. The OER tests were operated in a three-electrode cell with a working electrode $(W E)$ of glassy carbon flake $\left(10 \mathrm{~mm} \times 20 \mathrm{~mm} \times 0.5 \mathrm{~mm}\right.$; Effective electrode area: $\left.1.0 \mathrm{~cm}^{2}\right)$, a counter electrode of platinum foil, and a $\mathrm{Hg} / \mathrm{HgO}$ reference electrode (RE) (filled with $1 \mathrm{M} \mathrm{KOH}$ solution). The catalysts electrode was fabricated by the recipe drop-castes method which was reported in elsewhere ${ }^{2}$ The catalysts were mixed with acetylene black $(\mathrm{AB})$ at a mass ration of 5:1, then were dispersed in isopropanol/water $(\mathrm{v} / \mathrm{v}=1: 4)$ solvent followed by the addition of $\mathrm{Na}^{+}$-exchanged Nafion as the binder. The mixtures were ultrasonicated for $30 \mathrm{~min}$ to reach homogeneous ink. The concentration of oxides in ink is $5 \mathrm{mg} / \mathrm{ml}$, and $\mathrm{AB}$ is $1 \mathrm{mg} / \mathrm{ml}$. Before drop-casting, the glassy carbon electrodes were polished to a mirror finish with $\alpha-\mathrm{Al}_{2} \mathrm{O}_{3}(50 \mathrm{~nm})$ and washed by IPA and water to clean up completely. Finally, the asprepared ink (100 ul) was dropped onto glassy carbon electrodes to reach a loading mass of $500 \mu \mathrm{g}_{\mathrm{ox}} \mathrm{cm}^{-}$ 2 and the electrodes were dried overnight at room temperature. Cyclic voltammograms (CVs), linear sweep voltammetry (LSV) and chronoamperometry (CA) were performed in $\mathrm{O}_{2}$-saturated $1 \mathrm{M} \mathrm{KOH}$ by using Bio-logic SP 150 potentiostat. CA test under the different magnetic field strength $(0,500,1000$, $3000,5000,7500$, and $10000 \mathrm{Oe}$ ) at a constant potential of $1.66 \mathrm{~V}$ versus $\mathrm{RHE}$ for $\mathrm{CoFe}_{2} \mathrm{O}_{4}, \mathrm{Co}_{3} \mathrm{O}_{4}$, and $1.56 \mathrm{~V}$ versus $\mathrm{RHE}$ for $\mathrm{IrO}_{2}$. All potentials were converted to RHE scale according to the following equation: $\mathrm{RHE}=\mathrm{Hg} / \mathrm{HgO}+0.098$ with $\mathrm{iR}$ correction. The tests of methanol oxidation reaction (MOR) and ethylene glycol oxidation reaction (EGOR) on CFO electrodes are similar to the OER test. The difference is that the MOR and EGOR were studied in $1 \mathrm{M} \mathrm{KOH} 100 \mathrm{ml}$ electrolyte in the presence of 1 $\mathrm{ml}$ methanol and $1 \mathrm{ml}$ ethylene glycol, respectively ${ }^{3}$.

Materials characterization. The X-ray diffraction (XRD) of oxides were carried on Bruker D8 diffractometer at a scanning rate of $2^{\circ} \mathrm{min}^{-1}$, under $\mathrm{Cu}-\mathrm{K}_{\alpha}$ radiation $(\lambda=1.5418 \AA)$. DC magnetization 
measurements were performed on a Superconducting Quantum Design (SQUID) magnetometer (MPMSXL). The SQUID measurements of the magnetization of samples as a function of the magnetic field were carried out at $300 \mathrm{~K}$ in fields between $-5 \mathrm{~T}$ and $+5 \mathrm{~T}$. The high-resolution transmission electron microscopy (HRTEM) was carried JEOL JEM- 2100 plus microscope at 200KV. The STEM results presented here were obtained using the 200kV JEOL ARM electron microscope equipped (JEOL, Tokyo, Japan) with double aberration correctors, a dual-energy-loss spectrometer and a cold field emission source. The atomic-resolved STEM images were collected with a condense aperture of $28 \mathrm{mrad}$ and a collection angle of 90 - $370 \mathrm{mrad}$ for HAADF and $11-23 \mathrm{mrad}$ for ABF images. The XPS measurements were performed using PHI-5400 equipment with Al K $\alpha$ beam source $(250 \mathrm{~W})$ and a position-sensitive detector (PSD) was used to determine the surface composition of the materials. The Fourier transform infrared spectroscopy-Raman spectroscopy was carried with a confocal Raman microscope (Horiba HR Evolution), equipped with a diode laser emitting at $532 \mathrm{~nm}$. The nominal laser power was filtered down to $1 \mathrm{~mW}$ to avoid sample overheating. Spectra were recorded with the accumulation time of $60 \mathrm{~s}$. We have carried out HRTEM observation on the $\mathrm{CoFe}_{2} \mathrm{O}_{4}$ catalyst at the identical location before and after the electrochemical reaction using identical location TEM technique (IL-TEM). The detailed methods of IL-TEM are as follows. A diluted ink containing catalyst was pipetted onto the gold finder grid (400 mesh, TED PELLA, USA). The pristine catalyst on the gold finder grid was observed at the specific location before cycling. Then the grid was used at the working electrode in an electrochemical cell. After the electrochemical cycling, the grid was dried under Ar flow and observed under TEM again. The grid allowed us to find the particle at the same location as it was before electrochemical cycling. The images are shown in Supplementary Figure 11. It is clear that those particles remain unchanged after electrochemical cycling and there is no remarkable surface change.

DFT studies. All the density functional theory (DFT) calculations were performed by Vienna Ab-initio Simulation Package ${ }^{4,5}$ (VASP), employing the Projected Augmented Wave ${ }^{6}$ (PAW) method. The revised Perdew-Burke-Ernzerhof (RPBE) functional was used to describe the exchange and correlation effects. ${ }^{7-}$

${ }^{9}$ The GGA + U calculations are performed using the model proposed by Dudarev et al. ${ }^{10}$, with the $\mathrm{U}_{\text {eff }}$ $\left(\mathrm{U}_{\text {eff }}=\right.$ Coulomb $\mathrm{U}$ - exchange $\left.\mathrm{J}\right)$ values of $3.3 \mathrm{eV}$ and $4 \mathrm{eV}$ for $\mathrm{Co}$ and Fe, respectively. For all the geometry optimizations, the cutoff energy was set to be $500 \mathrm{eV}$. A $3 \times 3 \times 1$ Monkhorst-Pack grids ${ }^{11}$ was used to carry out the surface calculations on the (111) surface of $\mathrm{CoFe}_{2} \mathrm{O}_{4}$. At least $20 \AA$ vacuum layer 
was applied in z-direction of the slab models, preventing the vertical interactions between slabs.

In alkaline conditions, OER could occur in the following four elementary steps:
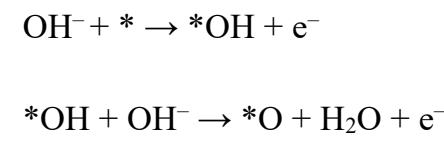

$* \mathrm{O}+\mathrm{OH}^{-} \rightarrow * \mathrm{OOH}+\mathrm{e}^{-}$

$* \mathrm{OOH}+\mathrm{OH}^{-} \rightarrow *+\mathrm{O}_{2}+\mathrm{H}_{2} \mathrm{O}+\mathrm{e}^{-}$

where * denotes the active sites on the catalyst surface. Based on the above mechanism, the free energy of three intermediate states, ${ }^{*} \mathrm{OH},{ }^{*} \mathrm{O}$, and ${ }^{*} \mathrm{OOH}$, are important to identify a given material's OER activity. The computational hydrogen electrode (CHE) model ${ }^{12}$ was used to address the electrochemical proton-electron transfer with applied potential. The free energies of each elementary step were defined as

$\Delta \mathrm{G}_{\mathrm{ads}}=\Delta \mathrm{E}_{\mathrm{ads}}+\Delta \mathrm{E}_{\mathrm{ZPE}}-\mathrm{T} \Delta \mathrm{S}_{\mathrm{ads}}$

where $\Delta E_{a d s}$ is the electronic adsorption energy, $\Delta E_{Z P E}$ is the zero point energy difference between adsorbed and gaseous species, and $T \Delta S_{a d s}$ is the corresponding entropy difference between these two states. The electronic binding energy is referenced as $1 / 2 \mathrm{H}_{2}$ for each $\mathrm{H}$ atom, and $\left(\mathrm{H}_{2} \mathrm{O}-\mathrm{H}_{2}\right)$ for each $\mathrm{O}$ atom, plus the energy of the clean slab. The corrections of zero point energy and entropy of the OER intermediates can be found in the Supplementary Table 2. The details of computational under magnetic field are as follows. The function of the outer magnetic field is to align all the randomly oriented spin in the catalyst to a specific direction. To model this situation, we have used the 'LNONCOLLINEAR' and 'SAXIS' keywords to make the spin in the catalyst to a specific direction. And during the calculations, we did not set initial guesses of the magnetic moments and let VASP to fully relax until finding out the most stable configuration. In the Supplementary Table 3, we have summarized the final magnetic moments $\left(\mu_{\mathrm{B}}\right)$ of the metal cations after structural optimization. The spin densities $\left(\mu_{\mathrm{B}}\right)$ of the adsorbed oxygen species summarized in Supplementary Table 4.

The surface Pourbaix diagram was calculated based on the method proposed by Hansen et al. ${ }^{13}$, where the free energy of oxygen and hydroxyl exchange at a given surface at any $\mathrm{pH}$ and potential is calculated as

$$
G(H O *)=\Delta G_{0}(H O *)-e U_{S H E}-k_{B} T \ln 10 p H+\Delta G_{\text {field }}
$$


110 Where $\Delta \mathrm{G}_{\text {field }}$ is the change in the adsorption energy due to the electric field in the electrochemical double

111 layer at the cathode. According to the work by Rossmeisl et al. ${ }^{14}$, the relative stability change of $\mathrm{O}^{*}$ and

$112 \mathrm{OH}^{*}$ under electric field is sufficiently low and it is believed that the trend in adsorption energies can be

113 well described by neglecting $\Delta \mathrm{G}_{\text {field }}$ in the construction of the surface Pourbaix diagram.

114 The term $\Delta \mathrm{G}_{0}$ is calculated by correcting the DFT energies for zero-point energies and entropy via

$$
\Delta G_{0}=\Delta E+\Delta Z P E-T \Delta S
$$

116 Where $\triangle \mathrm{E}$ and $\triangle \mathrm{ZPE}$ are the DFT energy and the change in zero-point energy of the adsorbates, respectively. More details of the calculation setup can be found in reference. ${ }^{13}$

118

119

120

121

122

123

124

125

126

127

128

129

130 

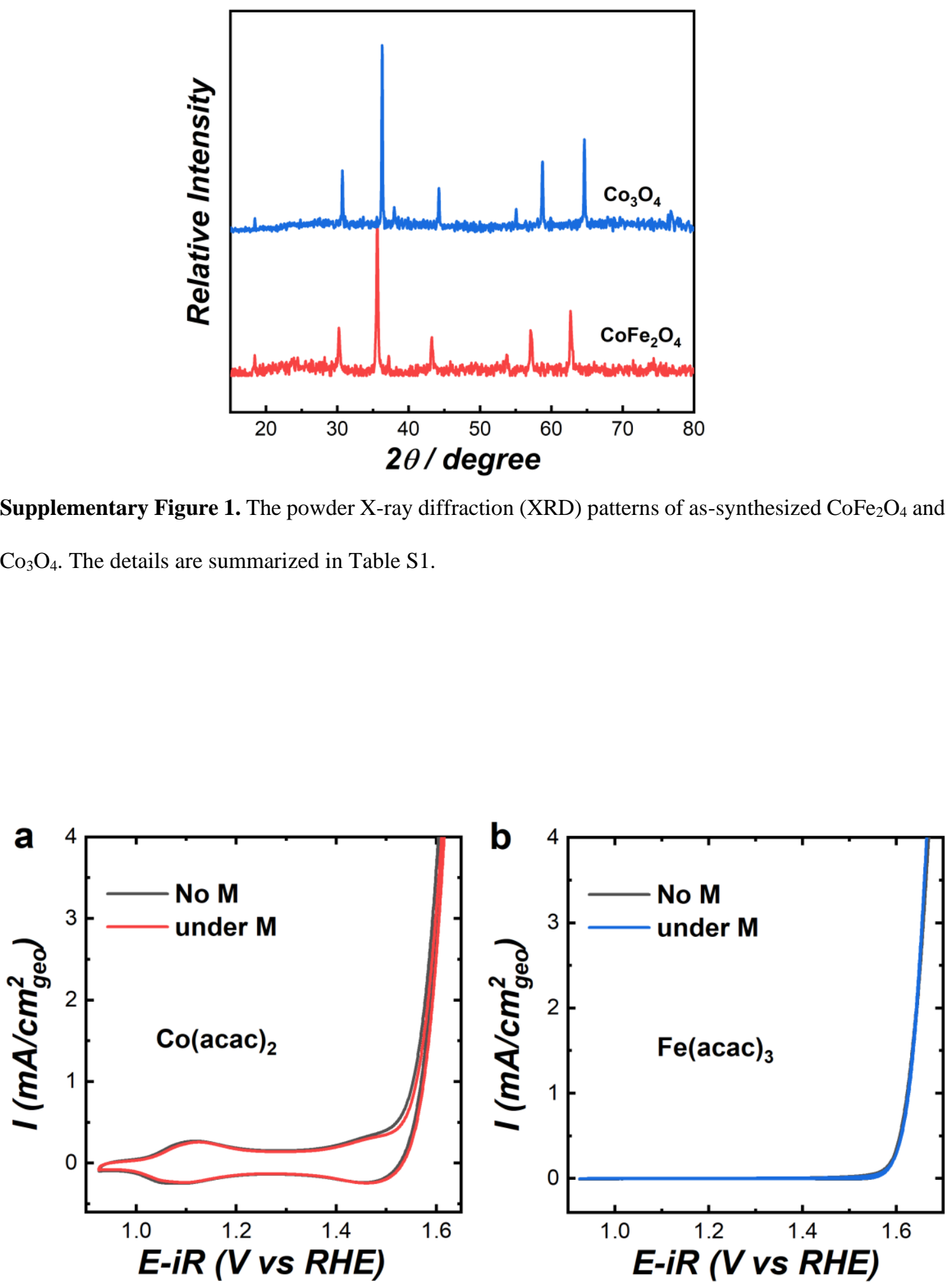

Supplementary Figure 2. Cyclic voltammetry $(\mathrm{CV})$ of $\mathrm{Co}(\mathrm{acac})_{2}$, (a) and $\mathrm{Fe}(\mathrm{acac})_{3}(\mathbf{b})$ catalysts at a scan rate of $10 \mathrm{mV} / \mathrm{s}$ in $\mathrm{O}_{2}$-saturated $1 \mathrm{M} \mathrm{KOH}$ with and without a constant magnetic field (10000 Oe). 
(a)

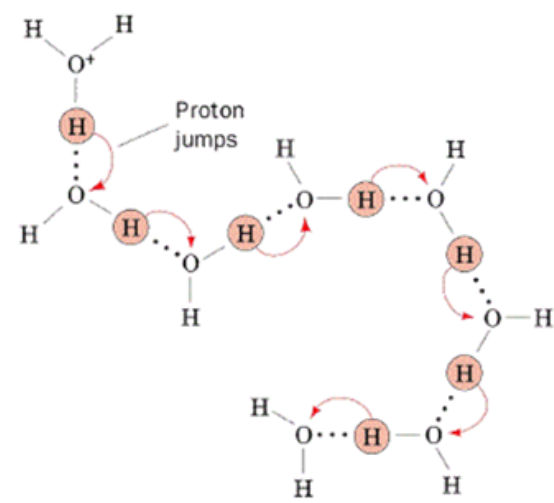

(b)

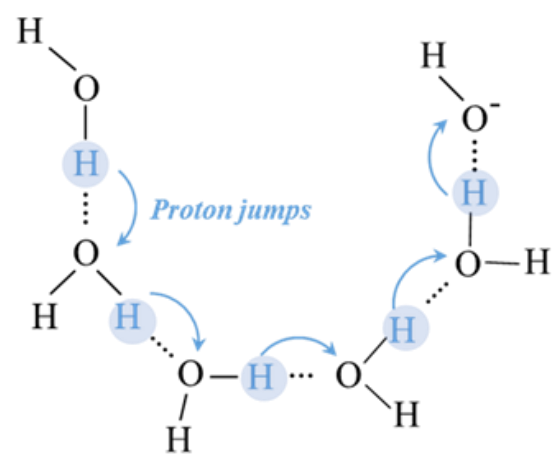

145

146

147

148

149

150

151

152

153

Supplementary Figure 3. The mechanism of proton hopping (jump) for (a) $\mathrm{H}_{3} \mathrm{O}^{+}$and (b) $\mathrm{OH}^{-}$in aqueous solution. ${ }^{15}$ 

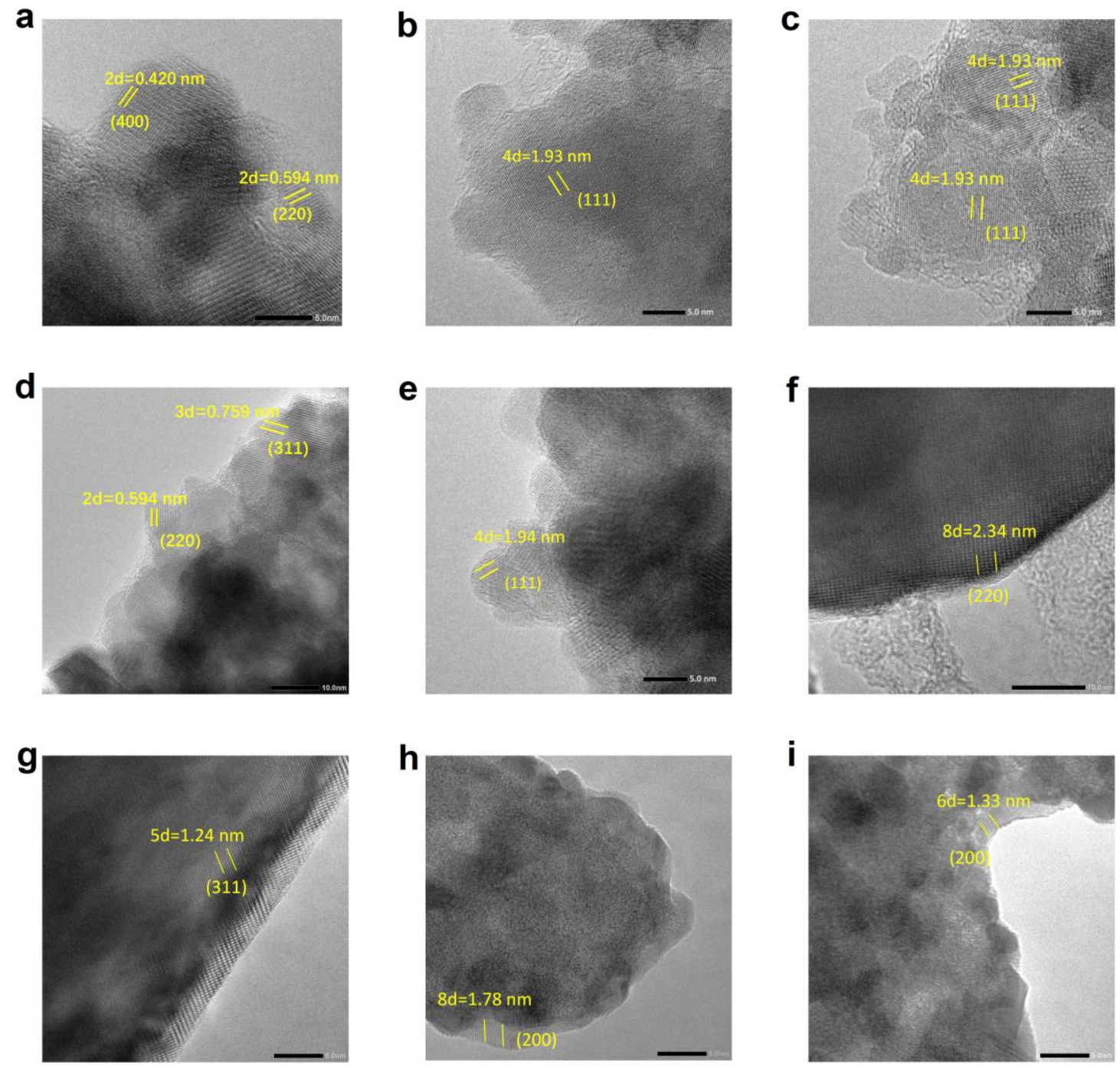

Supplementary Figure 5. HRTEM images of $\mathrm{CoFe}_{2} \mathrm{O}_{4}$ before (a-c) and after OER (d, e). HRTEM images of $\mathrm{Co}_{3} \mathrm{O}_{4}$ before (f) and after OER (g). HRTEM images of $\mathrm{IrO}_{2}$ before (h) and after OER (i). 


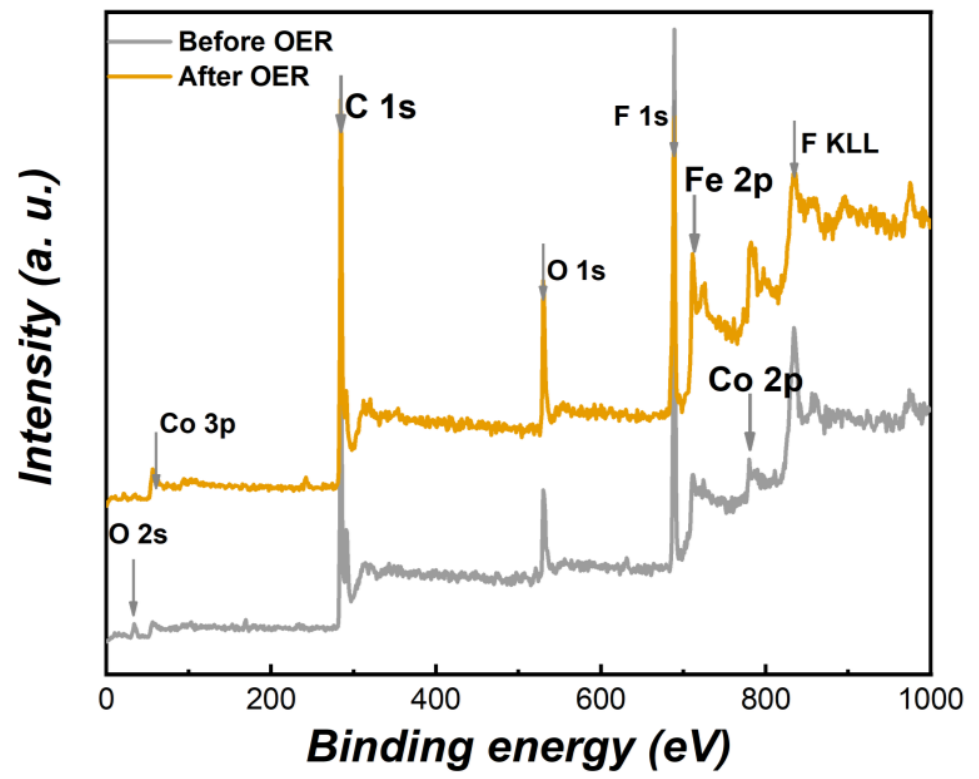

172

173 Supplementary Figure 6. The XPS survey spectra of $\mathrm{CoFe}_{2} \mathrm{O}_{4}$ before and after OER.

174

175

176

177

178

179

180

181

182

183

184

185

186 

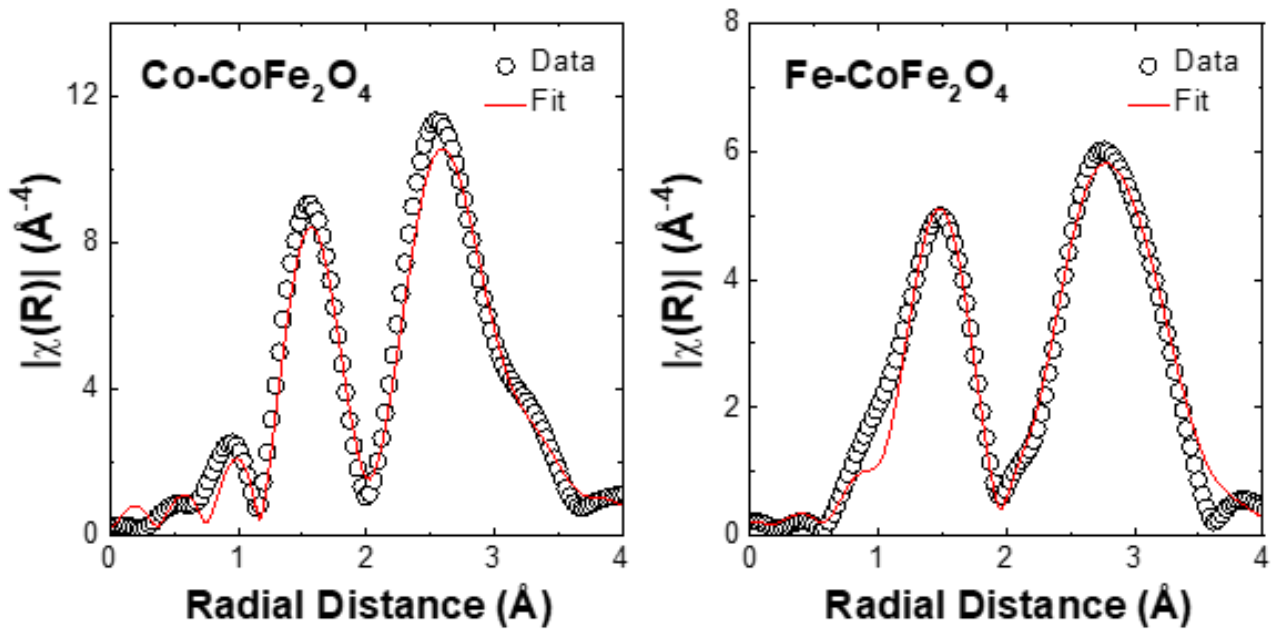

\begin{tabular}{|ccc|}
\hline & Tetrahedral site & Octahedral site \\
\hline $\mathrm{Co}_{\mathrm{x}}$ & 0.109 & 0.891 \\
\hline $\mathrm{Co}-\mathrm{O}(\AA)$ & 1.81 & 2.06 \\
$\mathrm{Co}-\mathrm{O}$ coordination No. & 3.3 & 4.9 \\
$\mathrm{Fe}_{\mathrm{y}}$ & 0.891 & 1.109 \\
\hline $\mathrm{Fe}-\mathrm{O}(\AA)$ & 1.93 & 1.99 \\
$\mathrm{Fe}-\mathrm{O}$ coordination No. & 3.0 & 4.5 \\
\hline & $\chi_{u}{ }^{2}=376.9 ; \mathrm{R}$ factor $=0.0188$ & \\
\hline
\end{tabular}

Supplementary Figure 7. EXAFS $\mathrm{k}^{3} \chi(\mathrm{R})$ spectra (gray circles) and fitting results (solid lines) of $\mathrm{CoFe}_{2} \mathrm{O}_{4}$ oxides at $\mathrm{Co}$ and $\mathrm{Fe} \mathrm{K}$-edge. The table show the summary of EXAFS fitting results for $\mathrm{CoFe}_{2} \mathrm{O}_{4}$. It can be seen that $\mathrm{CoFe}_{2} \mathrm{O}_{4}$ cubic spinels, $\approx 90 \%$ Co cations occupy octahedral. 


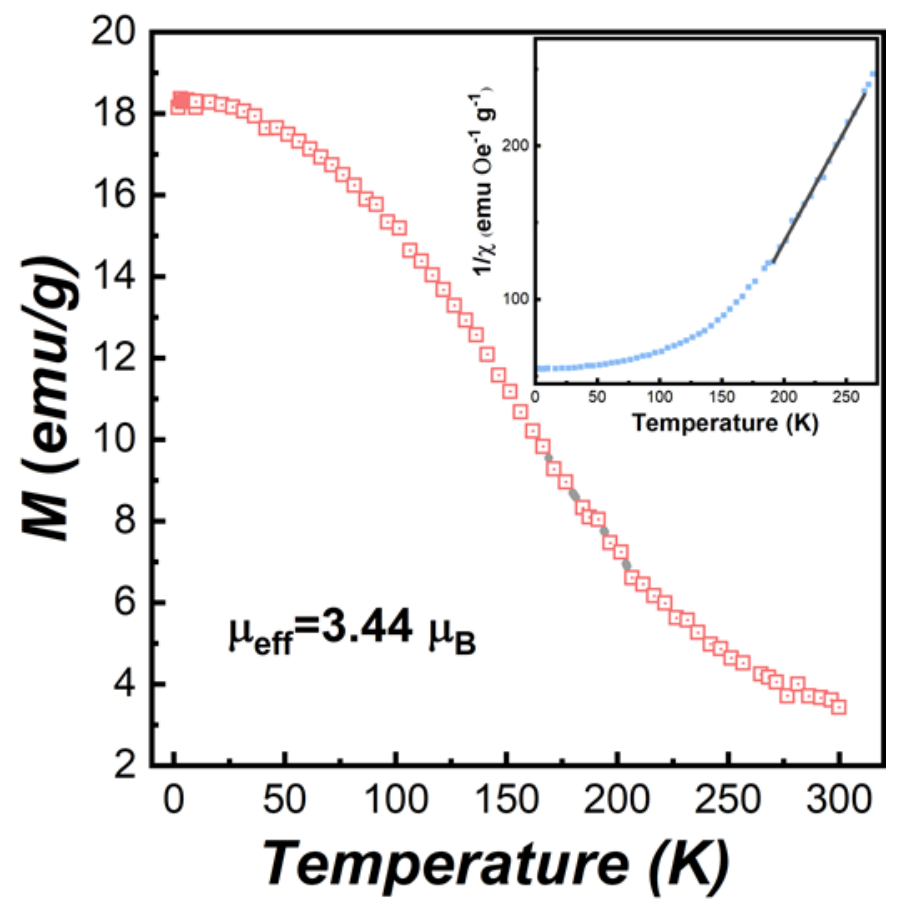

Supplementary Figure 8. The field-cooled M-T curves of $\mathrm{CoFe}_{2} \mathrm{O}_{4}$. The inset figure shows the temperature dependence of reciprocal susceptibilities. The solid line is the fitting results by the CurieWeiss law. In the high temperature area, the susceptibilities derived from the magnetizations $(\chi=M / H)$ obey a Curie-Weiss law: $\chi=C / T-T_{C}$, where $C$ is Curie constant, and $T_{C}$ is Curie-Weiss temperature. By fitting the susceptibility versus and $\mathrm{T}$ data, an effective magnetic moment $\mu_{e f f}$ can be obtained through $\mu_{e f f}=\sqrt{8 C} \mu_{B}$. Here, the calculated $\mu_{e f f}$ of $3.44 \mu_{\mathrm{B}}$ for the $\mathrm{CoFe}_{2} \mathrm{O}_{4}$ sample is very close to the idea inverse spinel value. 

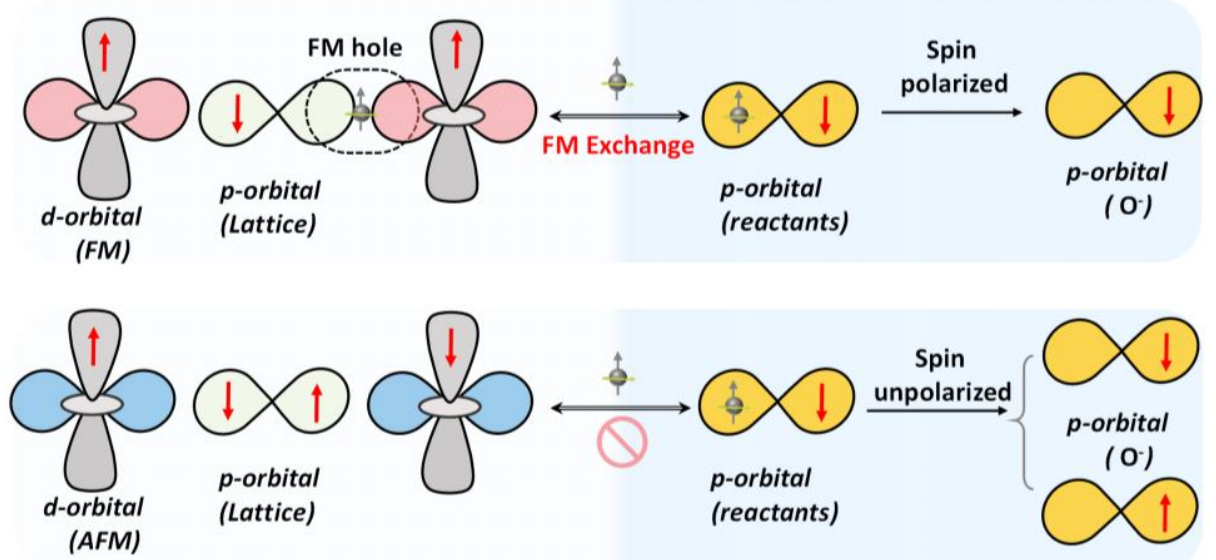

221

222

Supplementary Figure 9. Schematic diagram of spin electron transfer in a catalyst. FM holes present in the $\mathrm{M}-\mathrm{O}$ bonds and also in the oxygen ligands where the dominant FM exchange happens and enhances spin-selective charge transport.
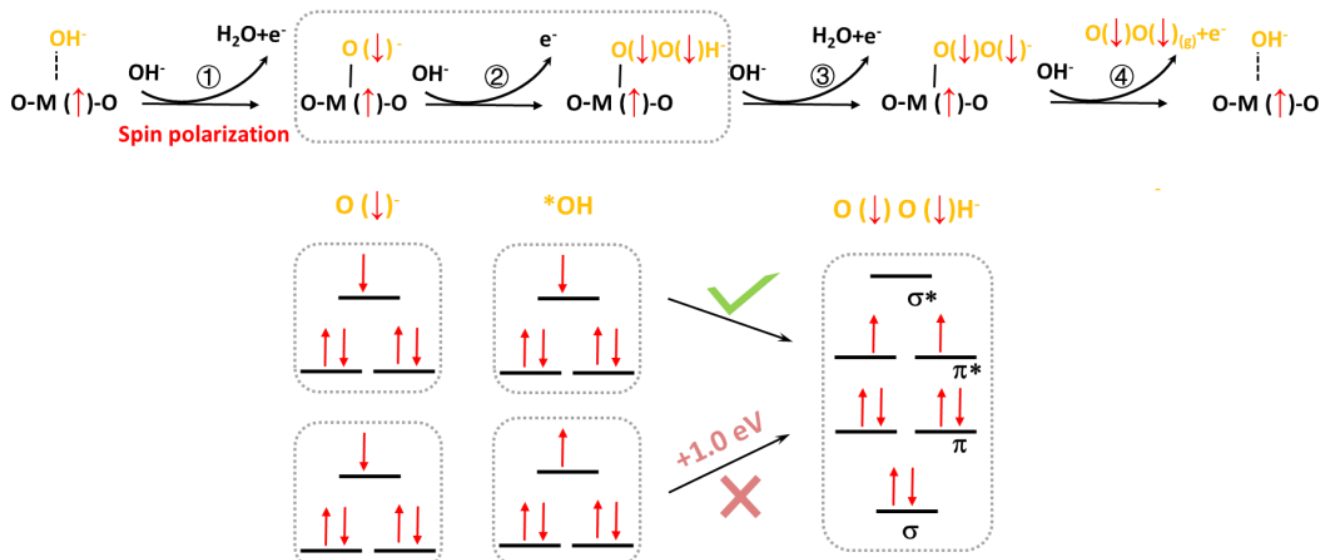

Supplementary Figure 10. The production of the triplet intermediate $O(\downarrow) O(\downarrow) H$ species. 

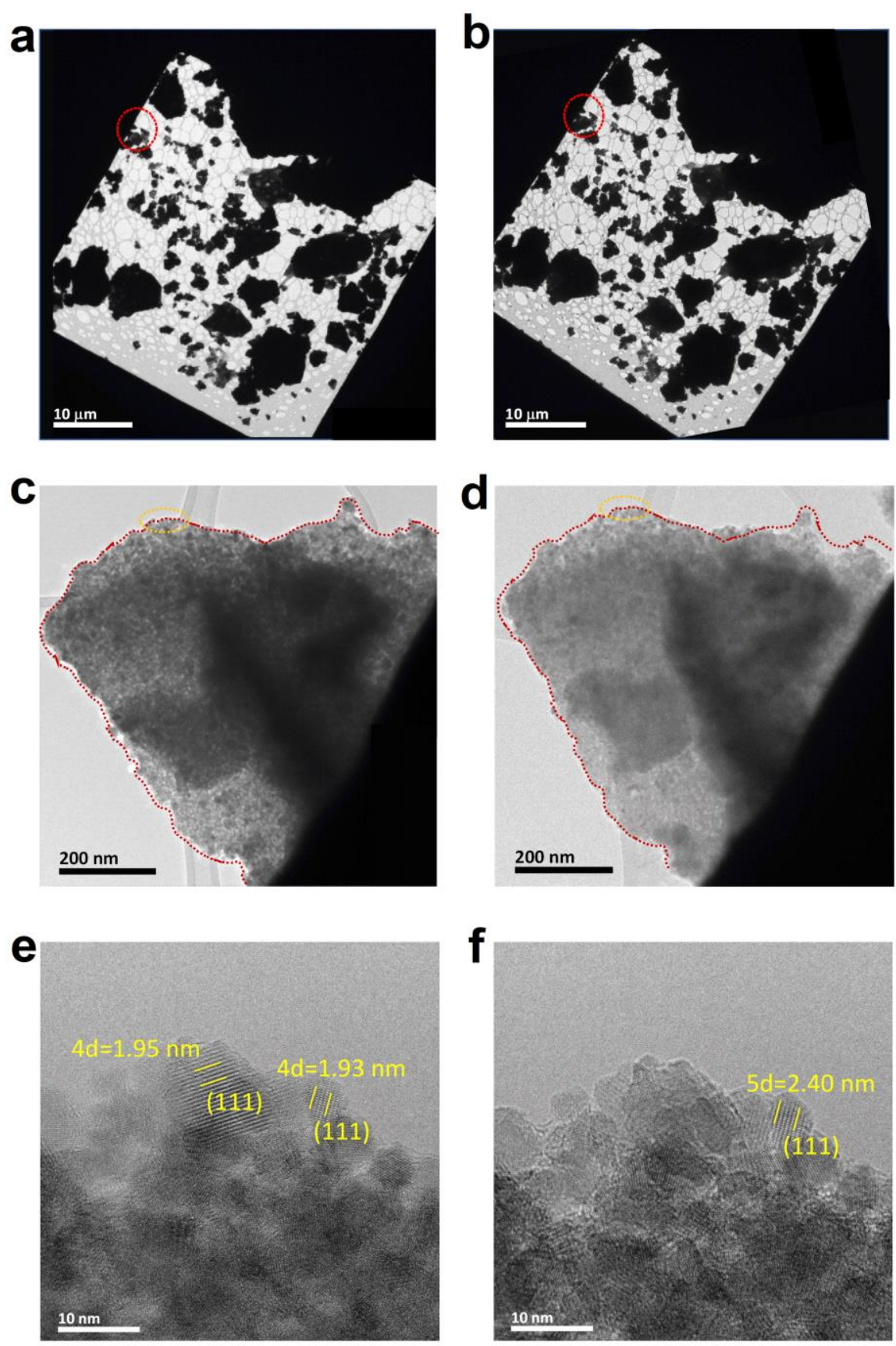

Supplementary Figure 11. Identical location TEM (IL-TEM) images of the $\mathrm{CoFe}_{2} \mathrm{O}_{4}$ before (a, c, and e) and after OER measurement (b, d, and $\mathbf{f})$. 


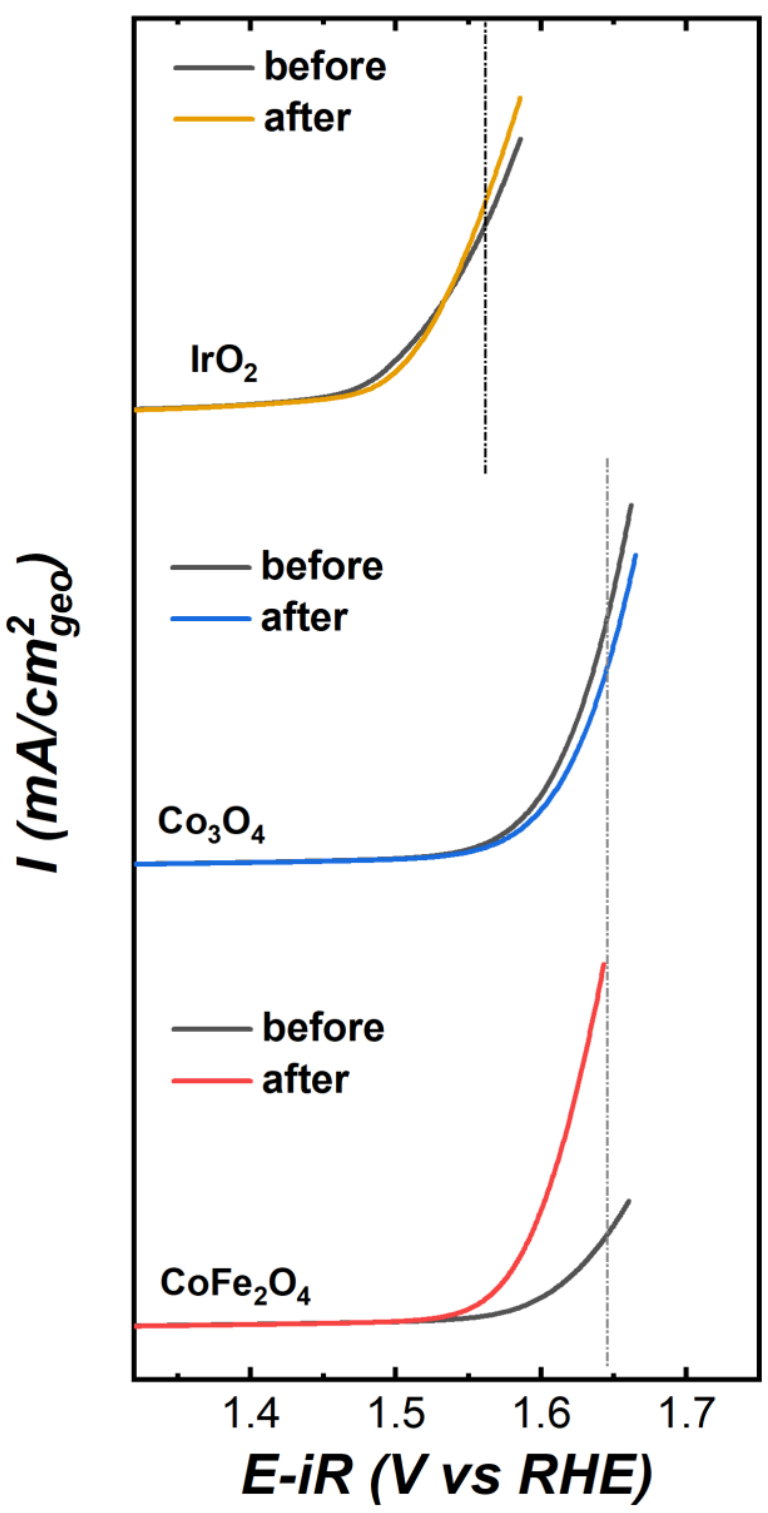
a scan rate of $10 \mathrm{mV} / \mathrm{s}$ in $\mathrm{O}_{2}$-saturated $1 \mathrm{M} \mathrm{KOH}$ before and after CA test. 

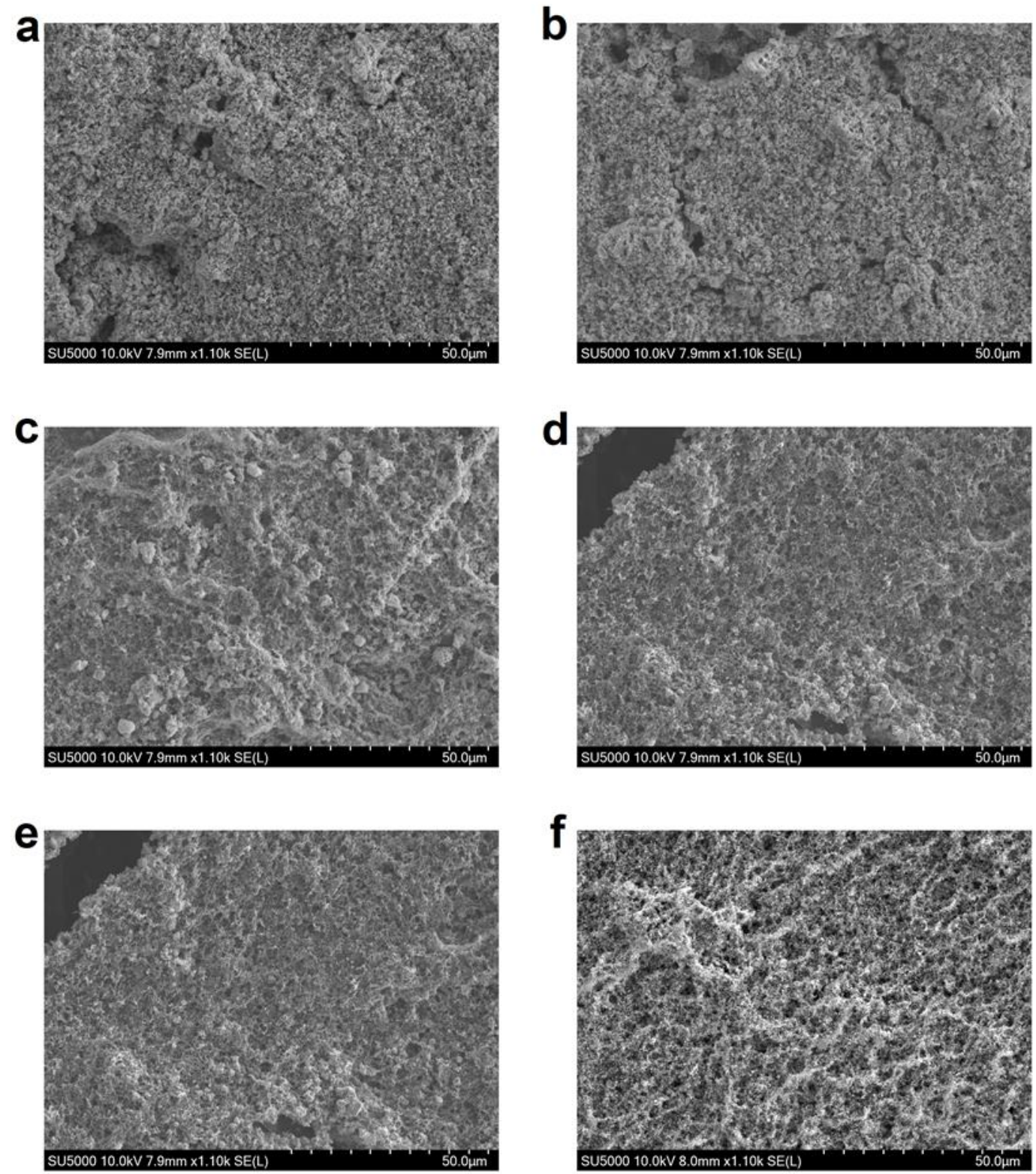

Supplementary Figure 13. SEM images of $\mathrm{CoFe}_{2} \mathrm{O}_{4}$ (a) before OER and (b) after OER; SEM images of $\mathrm{Co}_{3} \mathrm{O}_{4}$ (c) before OER and (d) after OER; SEM images of $\mathrm{IrO}_{2}(\mathbf{e})$ before OER and (f) after OER. As shown in Supplementary Figure, the microstructure of $\mathrm{CoFe}_{2} \mathrm{O}_{4}, \mathrm{Co}_{3} \mathrm{O}_{4}$, and $\mathrm{IrO}_{2}$ before and after OER test under magnetic field have no remarkable difference observed. 
Supplementary Table 1. Summary crystal structure parameters and magnetic data.

\begin{tabular}{|c|c|c|c|}
\hline Samples & Crystal structure & PDF & $\begin{array}{c}\text { Magnetism } \\
\text { (300K) }\end{array}$ \\
\hline $\mathrm{CoFe}_{2} \mathrm{O}_{4}$ & Spinel (F-3dm) & $22-1086$ & $\begin{array}{c}\text { Saturation } \\
\text { magnetization } \\
(\mathrm{Ms}): 44 \\
\text { emu/gCoercivity } \\
(\mathrm{Hc}): 887 \text { Oe }\end{array}$ \\
\hline $\mathrm{Co}_{3} \mathrm{O}_{4}$ & Spinel (F-3dm) & $42-1467$ & $\begin{array}{c}\text { Magnetic } \\
\text { susceptibility } \\
(\chi): 3.07 * 10-5\end{array}$ \\
\hline $\mathrm{IrO}_{2}$ & Rutile (P42/mnm) & $43-1019$ & $\chi: 0.51 * 10-6$ \\
\hline
\end{tabular}

272

273

274 275

\begin{tabular}{|c|c|c|}
\hline & ZPE (eV) & TS (eV) \\
\hline$* \mathrm{OOH}$ & 0.35 & 0 \\
\hline$* \mathrm{O}$ & 0.05 & 0 \\
\hline$* \mathrm{OH}$ & 0.31 & 0.01 \\
\hline $\mathrm{H}_{2} \mathrm{O}$ & 0.56 & 0.67 \\
\hline $\mathrm{H}_{2}$ & 0.27 & 0.41 \\
\hline
\end{tabular}

Supplementary Table 3. The magnetic moments $\left(\mu_{\mathrm{B}}\right)$ of the metal cations before and after structural optimization.

\begin{tabular}{|c|c|c|c|}
\hline & $\mathrm{Fe}_{(\mathrm{Tet})}$ & $\mathrm{Fe}_{(\mathrm{Oct})}$ & $\mathrm{Co}$ (Oct) \\
\hline Without aligned $\mathrm{CoFe}_{2} \mathrm{O}_{4}$ & 4.224 & 4.087 & 0.869 \\
\hline With aligned $\mathrm{CoFe}_{2} \mathrm{O}_{4}$ & 2.451 & 2.487 & 0.530 \\
\hline
\end{tabular}

Supplementary Table 4. Calculated spin densities $\left(\mu_{B}\right)$ of the adsorbed oxygen species.

\begin{tabular}{|c|c|c|c|}
\hline & $* \mathrm{OH}$ & $* \mathrm{O}$ & $* \mathrm{OOH}$ \\
\hline $\begin{array}{c}\text { Without spin } \\
\text { alignment }\end{array}$ & 0.065 & 0.195 & $\begin{array}{l}\mathrm{O} 1:-0.025 \\
\mathrm{O} 2:-0.063\end{array}$ \\
\hline $\begin{array}{c}\text { With spin } \\
\text { alignment }\end{array}$ & 0.053 & 0.091 & $\begin{array}{l}\mathrm{O} 1:-0.026 \\
\mathrm{O} 2:-0.044\end{array}$ \\
\hline
\end{tabular}




\section{References}

1. $\mathrm{Wu}, \mathrm{T}$. et al. Iron-facilitated dynamic active-site generation on spinel $\mathrm{CoAl}_{2} \mathrm{O}_{4}$ with selftermination of surface reconstruction for water oxidation. Nature Catalysis 2, 763-772 (2019).

2. Suntivich, J., Gasteiger, H.A., Yabuuchi, N. \& Shao-Horn, Y. Electrocatalytic Measurement Methodology of Oxide Catalysts Using a Thin-Film Rotating Disk Electrode. J. Electrochem. Soc. 157, B1263-B1268 (2010).

3. Sun, S., Zhou, Y., Hu, B., Zhang, Q. \& Xu, Z.J. Ethylene Glycol and Ethanol Oxidation on Spinel Ni-Co Oxides in Alkaline. J. Electrochem. Soc. 163, H99-H104 (2015).

4. Kresse, G. \& Furthmüller, J. Efficient iterative schemes for ab initio total-energy calculations using a plane-wave basis set. Phys. Rev. B 54, 11169-11186 (1996).

5. Kresse, G. \& Hafner, J. Ab initio molecular dynamics for liquid metals. Phys. Rev. B 49, 14251 14269 (1994).

6. Blöchl, P.E. Projector augmented-wave method. Phys. Rev. B 50, 17953-17979 (1994).

7. Perdew, J.P., Burke, K. \& Ernzerhof, M. Generalized Gradient Approximation Made Simple.

8. Zhang, Y. \& Yang, W. Comment on "Generalized Gradient Approximation Made Simple". Phys. Rev. Lett. 80, 890-890 (1998).

9. Hammer, B., Hansen, L.B. \& Nørskov, J.K. Improved adsorption energetics within densityfunctional theory using revised Perdew-Burke-Ernzerhof functionals. Phys. Rev. B 59, 74137421 (1999).

10. Dudarev, S., Botton, G., Savrasov, S., Humphreys, C. \& Sutton, A. Electron-energy-loss spectra and the structural stability of nickel oxide: An LSDA+ U study. Phys. Rev. B 57, 1505 (1998).

11. Monkhorst, H.J. \& Pack, J.D. Special points for Brillouin-zone integrations. Phys. Rev. B 13, 5188 (1976).

12. Nørskov, J.K. et al. Origin of the overpotential for oxygen reduction at a fuel-cell cathode. The Journal of Physical Chemistry B 108, 17886-17892 (2004).

13. Heine A. Hansen, J.R.a.J.K.N. Surface Pourbaix diagrams and oxygen reduction activity of Pt, Ag and Ni(111) surfaces studied by DFT. Phys. Chem. Chem. Phys. 10, 3722-3730 (2008).

14. Jan Rossmeisl, J.K.N., Christopher D. Taylor, Michael J. Janik, and Matthew Neurock Calculated Phase Diagrams for the Electrochemical Oxidation and Reduction of Water over Pt(111). J. Phys. Chem. B 110, 21833-21839 (2006).

15. Lee du, H., Choi, C.H., Choi, T.H., Sung, B.J. \& Kang, H. Asymmetric Transport Mechanisms of Hydronium and Hydroxide Ions in Amorphous Solid Water: Hydroxide Goes Brownian while Hydronium Hops. J.Phys. Chem. Lett. 5, 2568-2572 (2014). 